\title{
Experimental Results for the Multipath Performance of Galileo Signals Transmitted by GIOVE-A Satellite
}

\author{
Andrew Simsky, ${ }^{1}$ David Mertens, ${ }^{1}$ Jean-Marie Sleewaegen, ${ }^{1}$ Martin Hollreiser, ${ }^{2}$ and Massimo Crisci ${ }^{2}$ \\ ${ }^{1}$ Septentrio, Ubicenter, Philipssite 5, Leuven 3001, Belgium \\ 2 The European Space Research and Technology Centre, The European Space Agency, Keplerlaan 1, Postbus 299, \\ 2200 AG Noordwijk, The Netherlands
}

Correspondence should be addressed to Andrew Simsky, a.simsky@septentrio.com

Received 6 July 2007; Accepted 17 March 2008

Recommended by Olivier Julien

\begin{abstract}
Analysis of GIOVE-A signals is an important part of the in-orbit validation phase of the Galileo program. GIOVE-A transmits the ranging signals using all the code modulations currently foreseen for the future Galileo and provides a foretaste of their performance in real-life applications. Due to the use of advanced code modulations, the ranging signals of Galileo provide significant improvement of the multipath performance as compared to current GPS. In this paper, we summarize the results of about 1.5 years of observations using the data from four antenna sites. The analysis of the elevation dependence of averaged multipath errors and the multipath time series for static data indicate significant suppression of long-range multipath by the best Galileo codes. The E5AltBOC signal is confirmed to be a multipath suppression champion for all the data sets. According to the results of the observations, the Galileo signals can be classified into 3 groups: high-performance (E5AltBOC, L1A, E6A), mediumperformance (E6BC, E5a, E5b) and an L1BC signal, which has the lowest performance among Galileo signals, but is still better than GPS-CA. The car tests have demonstrated that for kinematic multipath the intersignal differences are a lot less pronounced. The phase multipath performance is also discussed.
\end{abstract}

Copyright (C) 2008 Andrew Simsky et al. This is an open access article distributed under the Creative Commons Attribution License, which permits unrestricted use, distribution, and reproduction in any medium, provided the original work is properly cited.

\section{INTRODUCTION}

The first Galileo signals were transmitted on January 12, 2006, by the GIOVE-A satellite. The first results for the tracking noise, signal power, and code multipath performance of the live GIOVE-A signal obtained with the use of Septentrio's GETR receiver have been presented in October 2006 [1]. The overview of the on-going GIOVE-A signal experimentation activity including results obtained at ESA, Septentrio NV, and Alcatel Alenia Space can be found in [2]. Results of GIOVE-A signal testing have also been reported in $[3,4]$.

The purpose of the current paper is to summarize the analysis of the multipath performance of the GIOVE-A signal performed at Septentrio since the beginning of the GIOVE-A mission up to the time of this publication that is during the first one and half years of the satellite operation. Estimations of code multipath errors specific to ranging signals are of particular interest to the user community because they make significant contribution to the error budget of user applications. Unlike many other error sources, multipath errors are essentially modulation-dependent, hence there is a significant interest to improving multipath performance by optimizing the signal definition.

The ranging signals of Galileo are based on advanced code modulation schemes, which are expected to provide significant improvement of the tracking and multipath performance as compared to the current GPS. With the advent of GIOVE-A these expectations have been verified. The first analysis [1] has clearly shown the advantages of the Galileo signals in comparison to current civilian signals of GPS (C/A and L2C). Further experience based on a wider array of data has confirmed these results. In this paper, we summarize the results from a number of data sets obtained at few antenna sites at different geographic locations as well as the results of kinematic tests in different environments.

The GIOVE-A transmits ranging signals using all the currently foreseen Galileo modulations: L1BC, L1A, E5a, E5b, E5 (or E5AltBOC), E6BC, and E6A $[1,5]$. The GETR receiver has been custom-built by Septentrio for the reception of GIOVE signals. The GETR is capable of tracking all the 


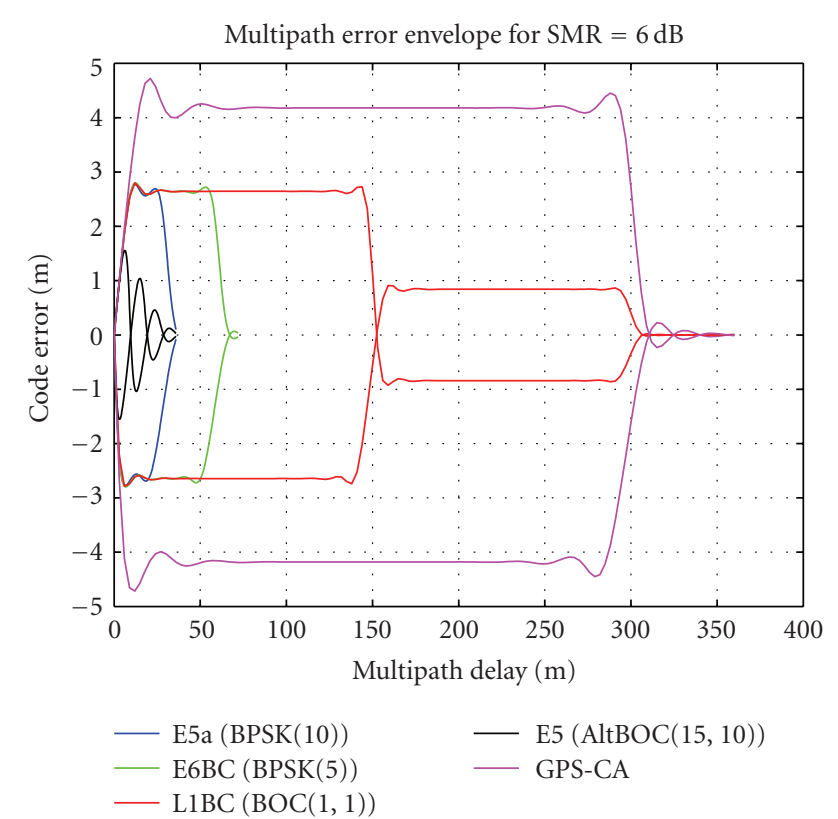

FIGURE 1: Multipath error envelopes of GNSS code modulation at signal/multipath ratio of $6 \mathrm{~dB}$ : GPS-C/A (magenta), Galileo L1BC (red), E6BC (green), E5a (blue), E5AltBOC (black).

transmitted modulations. The output of GETR includes raw measurements, navigation bits and, optionally, correlation function, and the samples of the RF signal at the intermediate frequency. The signal acquisition in GETR is implemented with the use of a custom-tailored fast acquisition unit [6].

This paper is based on the analysis of GETR measurements (pseudoranges, phases, Dopplers, $C / N_{0}$ ). The emphasis is on the evaluation of the code multipath performance, which is statistically characterized by the dependence of the averaged multipath noise upon elevation. Our approach is to compare empirical data for different sites and different signals and to classify the signals in accordance with their average multipath performance.

\section{MULTIPATH ERROR ENVELOPES OF GALILEO CODE MODULATIONS}

Multipath error envelopes for GPS-CA and Galileo code modulations are presented in Figure 1. The error envelopes were computed using the standard simulation of the tracking process of a straight code modulation superimposed with a single reflected signal at a signal/multipath ratio of $6 \mathrm{~dB}$. The simulation of the tracking process involves the computation of the correlation peaks of the original code and the code superimposed with multipath. The bandwidth of RF filtering simulated by the algorithm was $40 \mathrm{MHz}$ for all the codes ( $55 \mathrm{MHz}$ for E5AltBOC).

The results shown in Figure 1 prove that the error envelopes for all the Galileo modulations are well within the error envelope of the GPS-CA code. From the shape of the error envelopes it is evident that the biggest advantage of Galileo modulations is in the suppression of long-delay multipath. E5AltBOC is the only modulation, which is expected to provide a high degree of suppression of a short-range multipath as well. Exceptional multipath performance of E5AltBOC has been confirmed in all the hitherto processed data.

As for the other Galileo codes, their performance significantly depends upon the typical spectra of multipath delays on a particular site. For example, with a multipath delays of about $200 \mathrm{~m}$, the L1BC multipath is expected to be much lower than with GPS-CA, while with multipath delays of about $100 \mathrm{~m}$, the advantage of L1BC would be less pronounced. More precisely, the improvement of Galileo BOC $(1,1)$ with respect to $\operatorname{BPSK}(1)$ in the first $150 \mathrm{~m}$ is due to the wider transmit bandwidth of Galileo than GPS, and not really due to the signal structure. Indeed, if both Galileo and GPS had the same transmit BW, the multipath envelopes would be similar for the first $150 \mathrm{~m}$. The improvement due to signal structure only comes between 150 and $300 \mathrm{~m}$. All the Galileo codes presented in the plot (except for E5AltBOC) are expected to have the same multipath errors for delays shorter than $10 \mathrm{~m}$, while for the delays between 50 and $100 \mathrm{~m}$, E5a and E6BC modulations will have lower multipath errors than L1BC.

In practice, this means that relative performance of different code modulations will be site-dependent. Of course, a modulation with a smaller theoretical multipath error envelope is never expected to be worse (on average) than the modulation with a bigger error envelope. However, the advantages of more advanced code modulations will be more evident for the sites where long-delay multipath is dominant but may disappear for the sites with significant short-range mulitpath.

All the above considerations directly apply only to static multipath. Code multipath errors visible by the GNSS receiver in the movement, such as in car tests, are subject to averaging at the level of tracking. Although these modulations, which look better in Figure 1 are still expected to show lower multipath errors in the car test, it is hard to predict theoretically the measure of their advantage. Our experimental results presented later in the paper show relatively small differences between all the codes except for E5AltBOC which still is a definite champion. The exceptional qualities of E5AltBOC are due to its exceptionally high bandwidth. The tracking of E5AltBOC signal is implemented in the GETR in accordance with the algorithm outlined in [7].

\section{CALCULATION OF CODE MULTIPATH ERROR BASED ON EXPERIMENTAL DATA}

In our data analysis we computed code multipath using a well-known formula:

$$
M_{i}=P_{i}-\Phi_{i}+2 \lambda_{i}^{2} \frac{\Phi_{j}-\Phi_{i}}{\lambda_{j}^{2}-\lambda_{i}^{2}},
$$

where $M_{i}$ is the estimate of the code multipath error on a pseudorange $P_{i}$, while $\Phi_{i}$ and $\Phi_{j}$ are the carrier phase observables (in units of length) for wavelengths $\lambda_{i}$ and $\lambda_{j}$ for the same satellite. $j$ represents any band which is different 


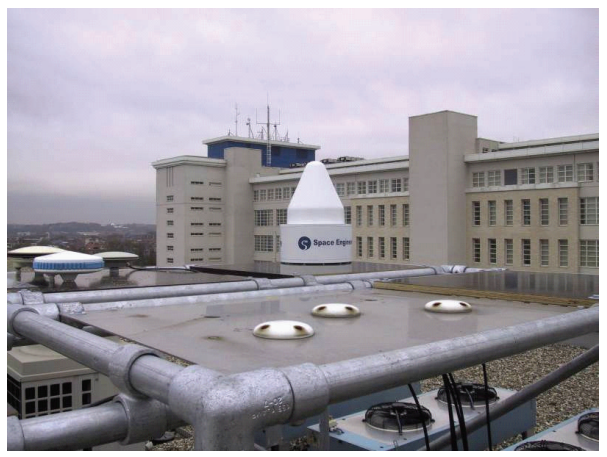

FIGURE 2: Space Engineering antenna mounted on the rooftop of the Septentrio office.

than $i$. With multifrequency Galileo signals, several values of $j$ are possible, but the particular selection of $j$ does not significantly affect the results. Formula (1) estimates a combination of multipath and tracking noise, but the contribution of the tracking noise can be neglected in most practical cases. For those signals which have pilot and data components, we used the pilot component; the multipath is exactly the same for both components but the tracking noise is independent. In (1), all the effects of the movement are canceled out, hence it is applicable to both static and kinematic data.

\section{STATIC DATA COLLECTED IN LEUVEN AT SEPTENTRIO TEST SITE}

Most of the data presented in this paper have been collected at the rooftop of the Septentrio office building. The wideband GPS/Galileo antenna provided by Space Engineering is shown in Figure 2. The antenna was mounted on the support structure and was located higher than the other objects on the rooftop. However, the adjacent building, which is seen at the photo, was still higher than the antenna and acted as a source of reflected signals. Therefore, the shortrange multipath at our site is relatively low, but long-range multipath systematically affects our data especially at low elevations when a satellite is rising or setting in the direction opposite to the adjacent building, which was in fact quite typical for GIOVE. The reflector building stretches in the North/South direction while GIOVE-A would often (but not always) rise directly in the East. In fact, day-dependent variations of multipath on our site were to a great extent due to the variations of the direction of rising/setting of GIOVEA with respect to this reflecting wall.

Table 1 shows the availability of the data for individual Galileo signals in the Leuven data sets processed for this report. Although GIOVE-A is able of transmitting all the experimental Galileo signals, it can transmit only in two frequency bands at a time. In reality, the satellite is transmitting either a combination of $\mathrm{L} 1+\mathrm{E} 5 \mathrm{a}+\mathrm{E} 5 \mathrm{~b}$ or a combination of L1+E6.

In our analysis, we have joined all the processed data for averaged signal power and code multipath errors as functions of elevation into one global array. This data is presented in

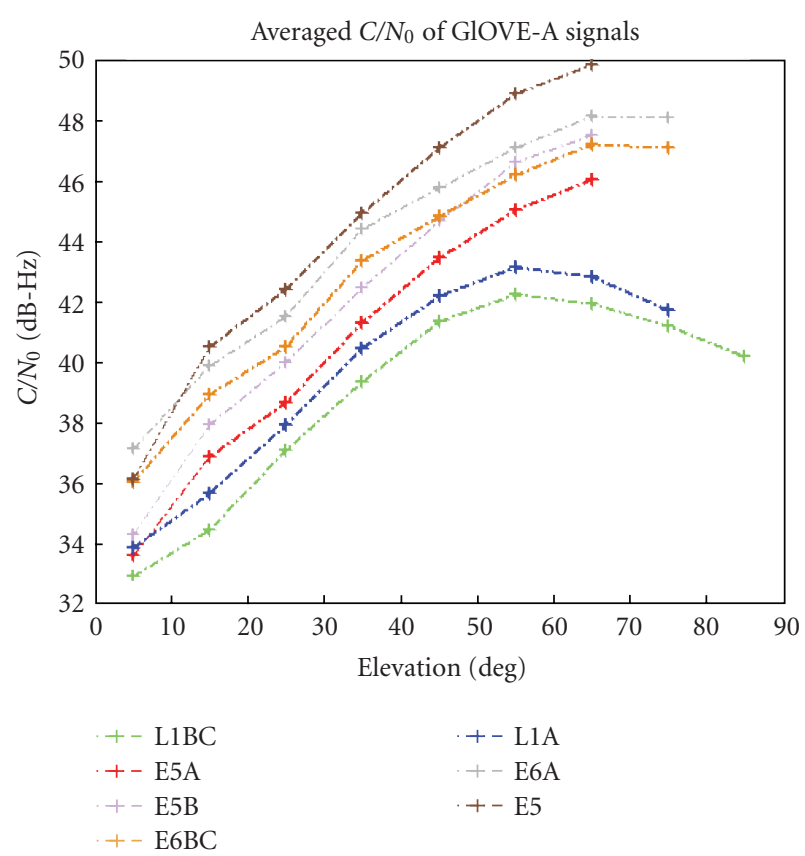

FIgURE 3: Averaged signal power for all the tests in Leuven.

Figures 3 and 4 for signal power and multipath, respectively. The signal power matches the specifications of GSTB-V2, but it is not representative of the final Galileo satellites, which will use different transmitters. The drop of $C / N_{0}$ at zenith for L1 signals is peculiar to the Space Engineering antenna (see [1] for more details).

Figure 4 contains standard deviations of code multipath for 10-degree bins of the elevation angle. Because the distance to the adjacent building is about $100 \mathrm{~m}$, typical delays of generated multipath are about $200 \mathrm{~m}$ (for satellites rising or setting in the direction opposite to the building), hence at low elevations L1BC and all the other Galileo codes perform significantly better than GPS-C/A, where this component of multipath is dominant. On the contrary, at high elevations where short-range multipath is dominating, GPS-CA and 4 Galileo codes (L1-BC, E5a, E5b, E6BC) have similar values of multipath errors.

It is also clear that at low elevations L1BC has the highest multipath compared to other Galileo modulations. For the best modulations, such as E5AltBOC and L1A the long-range multipath is almost completely suppressed, hence corresponding curves in Figure 4 are almost flat and show little increase at low elevations. The Leuven site is wellsuited to compare the suppression of long-range multipath by different code modulations.

Figure 4 contains also the comparison of the multipath performance of GPS-CA for 2 GPS receivers: GETR and PolaRx2. The difference between the two (black curves) can be seen as a measure of difference between the magnitude of multipath errors in two different receivers even if both do not use multipath mitigation (PolaRx2 uses multipath mitigation by default but it was turned off for this test). The difference 
TABLE 1: GIOVE-A signal components recorded during static tests in Leuven.

\begin{tabular}{|c|c|c|c|c|c|c|c|c|}
\hline Data set & Max elevation (deg) & L1A & L1BC & E5A & E5B & E5AltBoc & E6A & E6BC \\
\hline 15 January 2006 & 44.8 & $\mathrm{X}$ & $\mathrm{x}$ & & & & $\mathrm{x}$ & $\mathrm{x}$ \\
\hline 16 January 2006 & 60.0 & $\mathrm{X}$ & $\mathrm{x}$ & $\mathrm{x}$ & $\mathrm{x}$ & $\mathrm{x}$ & & \\
\hline 08 March 2006 & 83.8 & $\mathrm{X}$ & $\mathrm{x}$ & & & & $\mathrm{x}$ & $\mathrm{x}$ \\
\hline 19 May 2006 & 65.3 & $\mathrm{X}$ & $\mathrm{x}$ & $\mathrm{x}$ & $\mathrm{x}$ & $\mathrm{x}$ & & \\
\hline 28 May 2006 & 84.4 & & $\mathrm{x}$ & $\mathrm{x}$ & $\mathrm{x}$ & $\mathrm{x}$ & & \\
\hline 13 October 2006 & 75.6 & $\mathrm{X}$ & $\mathrm{x}$ & & & & $\mathrm{x}$ & $\mathrm{x}$ \\
\hline
\end{tabular}

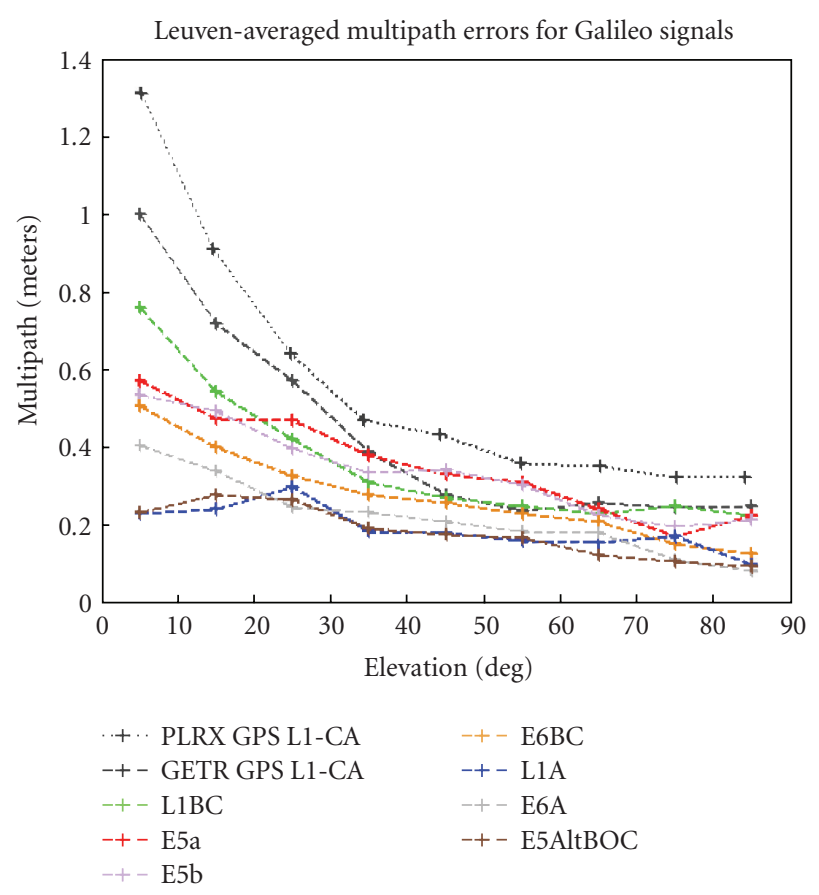

FIGURE 4: STD of code multipath for Galileo signals in comparison to GPS-CA for the tests in Leuven.

is due to a combination of receiver parameters such as frontend bandwidth and the type of a discriminator.

Comparison of low-elevation and high-elevation multipaths is also presented in Table 2. In this table, the Galileo modulations are grouped into 3 groups: (i) highperformance group, which included E5AltBOC and the two PRS modulations (L1A and E6A), (ii) medium-performance group, which includes E5a, E5b, and E6BC, and (iii) lowperformance group, which includes only L1BC and has still better performance as compared to CPS-CA. The values of multipath typical for the high-performance group are comparable to the values of tracking noise for GPS-CA code and are for most of the tests nearly equal at low and high elevations. This ranking of Galileo modulations in terms of multipath performance is practically identical to the ranking obtained by computer simulations in [8].

Successful suppression of long-range multipath can also be directly observed in the time series of multipath which we present here for some of the tests.

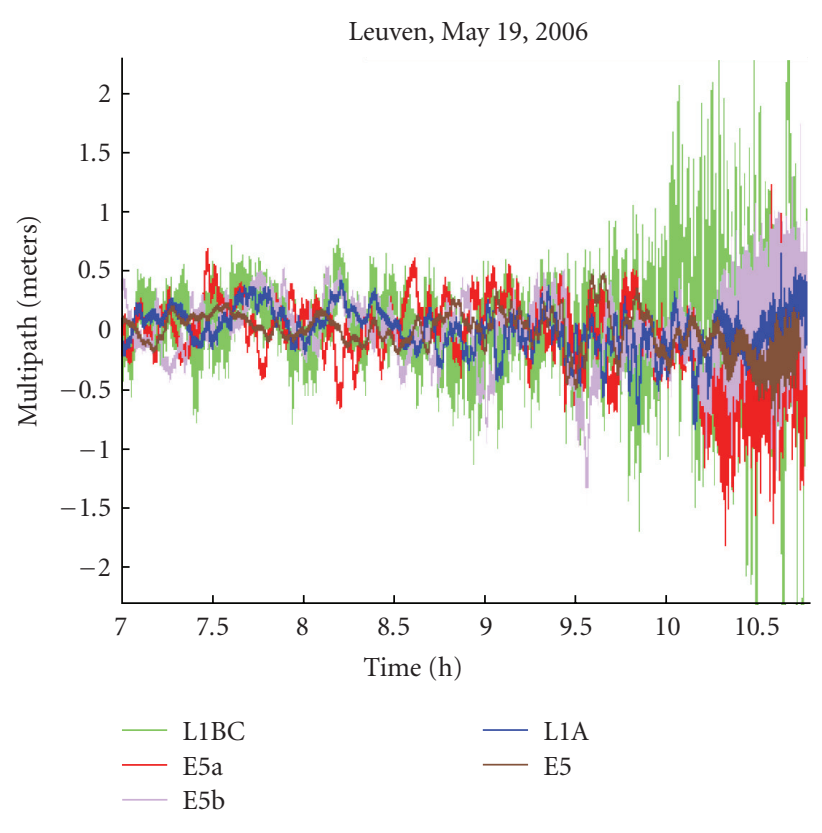

Figure 5: Time series of code multipath for the test of May 19.

In Figure 5, the long-range multipath manifests itself in high-frequency variations of multipath error near the right edge of the graph. The same ranking of the Galileo modulations as in Table 2 can be observed; the multipath errors of L1BC are the highest, while the multipath of E5AltBOC is the lowest and the others fall in-between.

The high-amplitude high-frequency variations of L1BC multipath shown in Figure 5 and other similar plots correspond in fact to a quasiperiod about 20 seconds. The zoomed plot of these variations is shown in Figure 6. This plot clearly demonstrates how complete the suppression of long-range multipath by the best Galileo modulations is. A similar example, which includes E6A, is shown in Figure 7. In Figure 5 and other plots with time series, the part of the plot with higher-amplitude and higher-frequency multipath always corresponds to lower elevations, when the satellite is rising and setting. The variation of multipath with elevation is illustrated by the multipath versus elevation plots (Figure 4 and similar plots). 
TABLE 2: Multipath STD error (m) of Galileo signals as compared to GPS C/A code.

\begin{tabular}{lcccccccccccccc}
\hline \multirow{2}{*}{ Signal } & Chip rate, & \multicolumn{2}{c}{ Jan. 15 } & \multicolumn{3}{c}{ Jan. 16} & \multicolumn{3}{c}{ Mar. 08 } & \multicolumn{2}{c}{ May. 19 } & \multicolumn{2}{c}{ May. 28 } & Oct. 13 \\
& $\mathrm{MHz}$ & $>10^{\circ}$ & $<10^{\circ}$ & $>10^{\circ}$ & $<10^{\circ}$ & $>10^{\circ}$ & $<10^{\circ}$ & $>10^{\circ}$ & $<10^{\circ}$ & $>10^{\circ}$ & $<10^{\circ}$ & $>10^{\circ}$ & $<10^{\circ}$ \\
\hline GPS-C/A & 1.023 & 0.60 & 1.19 & $*$ & $*$ & $*$ & $*$ & $*$ & $*$ & $*$ & $*$ & $*$ & $*$ \\
\hline L1BC & 1.023 & 0.36 & 0.93 & 0.40 & 0.55 & 0.39 & 0.41 & 0.38 & 0.86 & 0.38 & 0.93 & 0.34 & 0.79 \\
\hline E5a & 10.23 & - & - & 0.55 & 0.62 & - & - & 0.33 & 0.51 & 0.25 & 0.51 & - & - \\
E5b & 10.23 & - & - & 0.33 & 0.44 & - & - & 0.35 & 0.47 & 0.27 & 0.55 & - & - \\
E6BC & 5.115 & 0.28 & 0.28 & - & - & 0.27 & 0.42 & - & - & - & - & 0.30 & 0.67 \\
\hline L1A & 2.5575 & 0.24 & 0.37 & 0.21 & 0.20 & 0.24 & 0.20 & 0.23 & 0.22 & - & - & 0.18 & 0.37 \\
E6A & 5.115 & 0.24 & 0.22 & - & - & 0.23 & 0.17 & - & - & - & - & 0.23 & 0.58 \\
E5AltBOC & 10.23 & - & - & 0.25 & 0.23 & - & - & 0.20 & 0.30 & 0.14 & 0.23 & - & - \\
\hline
\end{tabular}

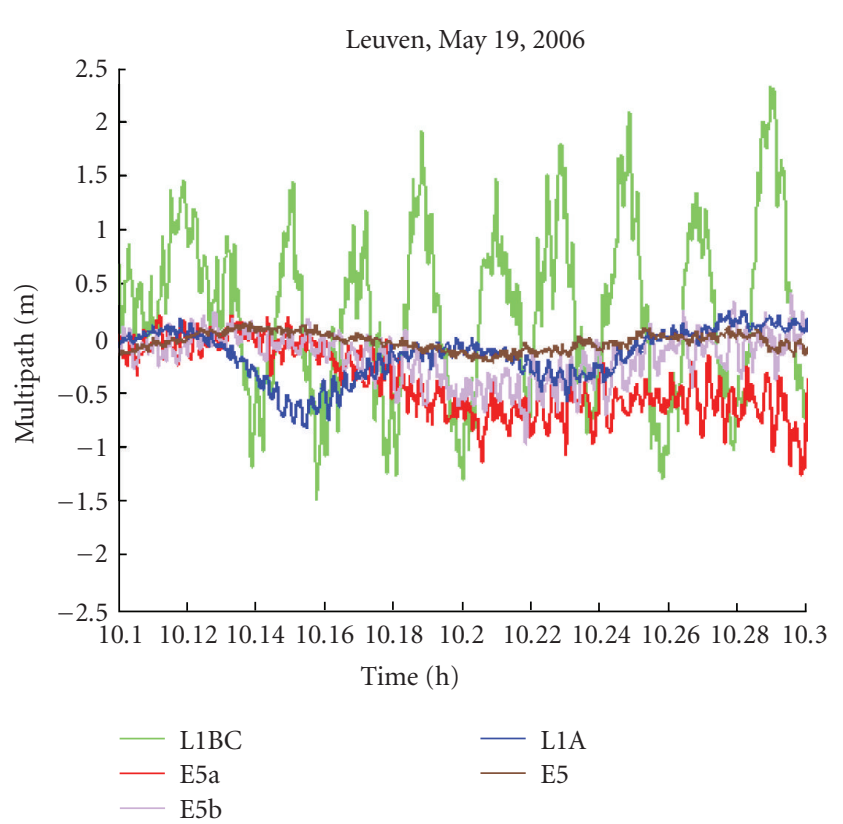

FIgURE 6: Zoomed view of the high-elevation part of the previous plot.

Although most of the Leuven data demonstrate similar behavior for all the 3 modulations of the best group (E5, L1A, E6A), a more careful analysis gives the impression that on average the magnitude of multipath errors increases in the sequence $\mathrm{E} 5 \mathrm{AltBOC} \rightarrow \mathrm{L} 1 \mathrm{~A} \rightarrow \mathrm{E} 6 \mathrm{~A}$ (which is quite in line with theoretical expectations), and that the performance of E6A in some cases comes close to the values typical for the "medium-performance" group. An example is presented in Figure 8. In fact, even in a summary plot (Figure 4), the E6A modulation shows visibly higher multipath errors at low elevations than E5AltBOC+L1A. Some other tests presented later in this paper also suggest that the bestperformance group in fact includes only E5AltBOC and L1A, while E6A gravitates to the medium-performance group. The E5AltBOC, on the other hand, always shows an exceptionally stable performance; its values of multipath errors are always the lowest as compared to other modulations (see Figures 9, 10 , and 11)

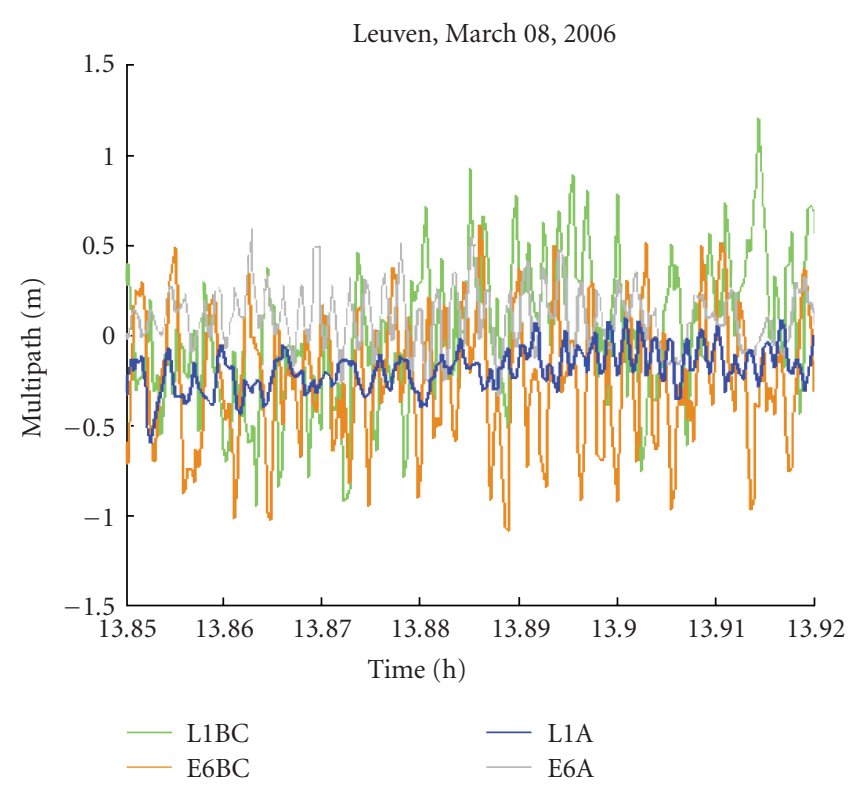

FIgURE 7: Similar example from another dataset which includes E6A.

\section{ANTENNA SITE IN LEUVEN WITH MORE INTENSIVE SHORT-RANGE MULTIPATH}

In order to investigate the effect of short-range multipath on Galileo signals, we placed the Galileo antenna at another more multipath-rich position on the same rooftop. This antenna position was located on the roof floor between the two metal ventilation outlets (identical to these in the right bottom corner of Figure 2). The antenna was located lower than many other reflective objects on the rooftop, so it was expected to get more short-range and middle-range multipath compared to the main site. The comparison of the two sites is presented in Figure 12.

Figure 12 shows that the multipath at the "multipathrich" location is indeed higher, the difference being particularly great for E5a. At higher elevations the difference between the two sites is statistically insignificant, which indicates that the local objects generate multipath predominantly 


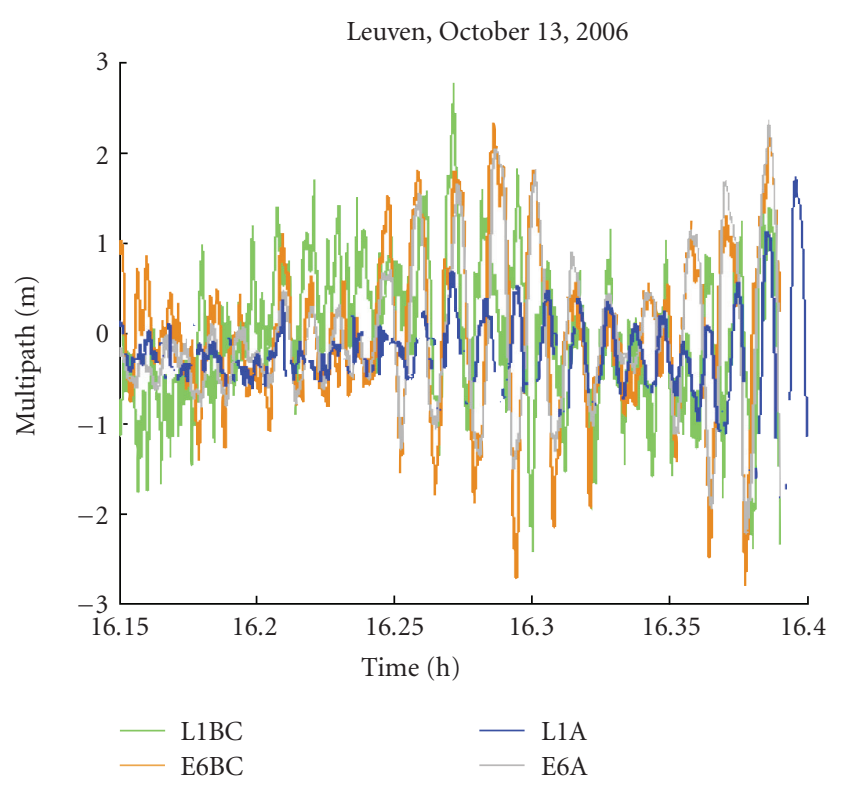

FIGURE 8: An occasion of relatively high multipath errors by L1A/E6A.

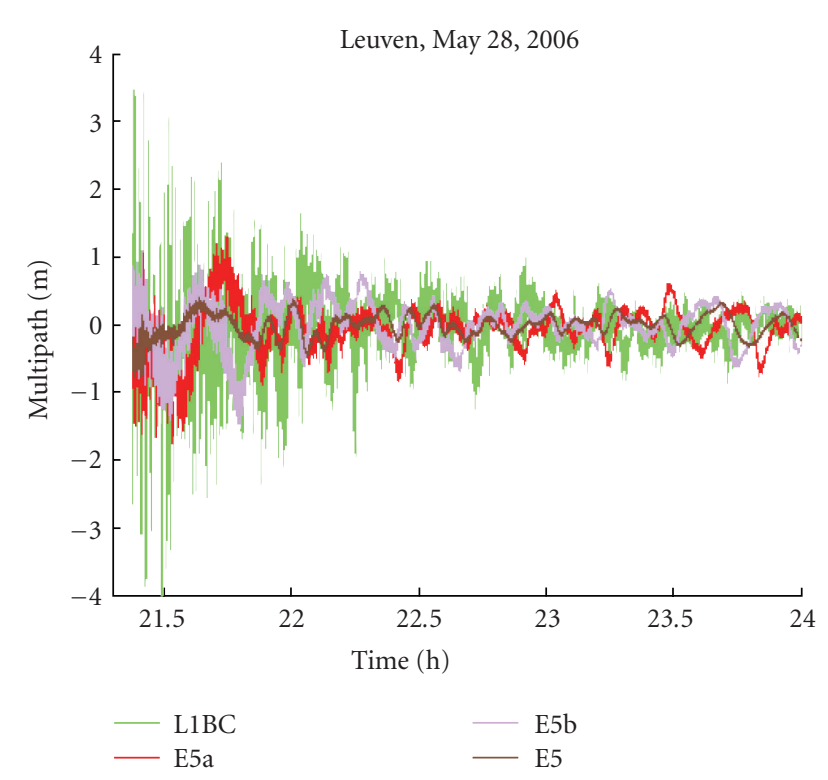

FIGURE 9: Multipath time series for May 28, 2006.

for low-elevation satellites. The multipath statistics for the two tests at the "multipath-rich" site is presented in Table 3.

The time series of code multipath is presented in Figures 13 and 14. It is evident that in both plots the multipath of E5a is unusually high in comparison to all the other tests. The reason for this strange behavior, different from all the other tests, is not clear.

\section{STATIC DATA COLLECTED AT LA PLATA AND WUHAN GESS SITES}

On top of processing the data collected by ourselves, we also processed the GIOVE-A data collected at 2 other geographic

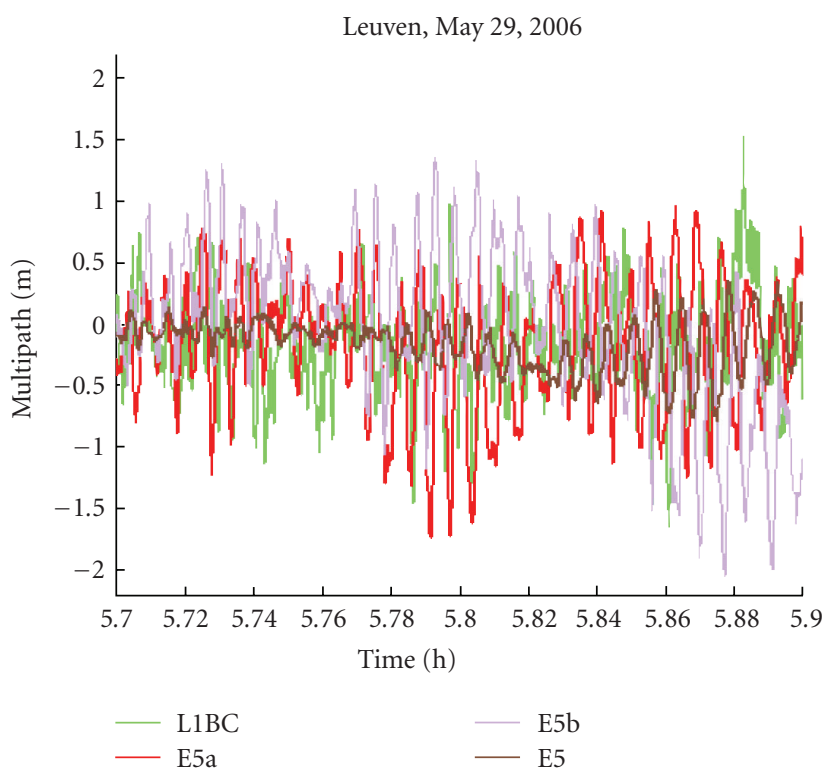

Figure 10: Multipath time series for May 29, 2006.

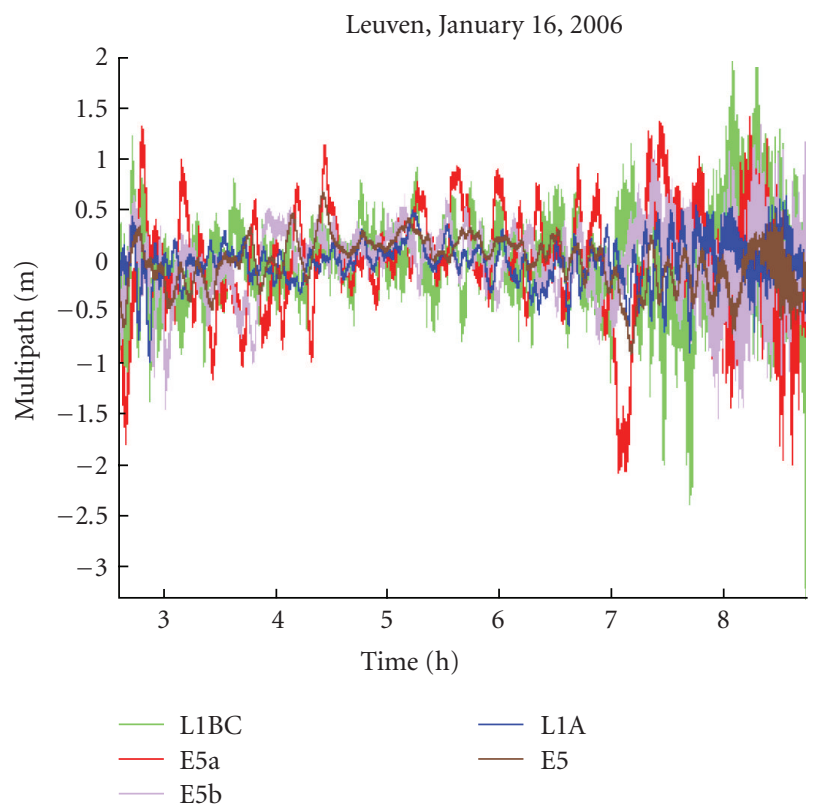

FIGURE 11: Multipath time series for January 16, 2006.

locations and available via GESS network: La Plata in Latin America and Wuhan in China.

The analysis of multipath data from these two sites confirms in broad terms the tendencies reported in the first section. In particular, the superior performance of L1A and E5AltBOC has been confirmed. However, some important differences must be mentioned. First of all, the E6BC signal has high multipath comparable to L1BC (even higher than L1BC at low elevations). Secondly, at the Wuhan site the elevation dependence is much less pronounced than for the rest of the tests, probably due to the peculiarities of local reflectors. Thirdly, the E6A signal shows worse performance 


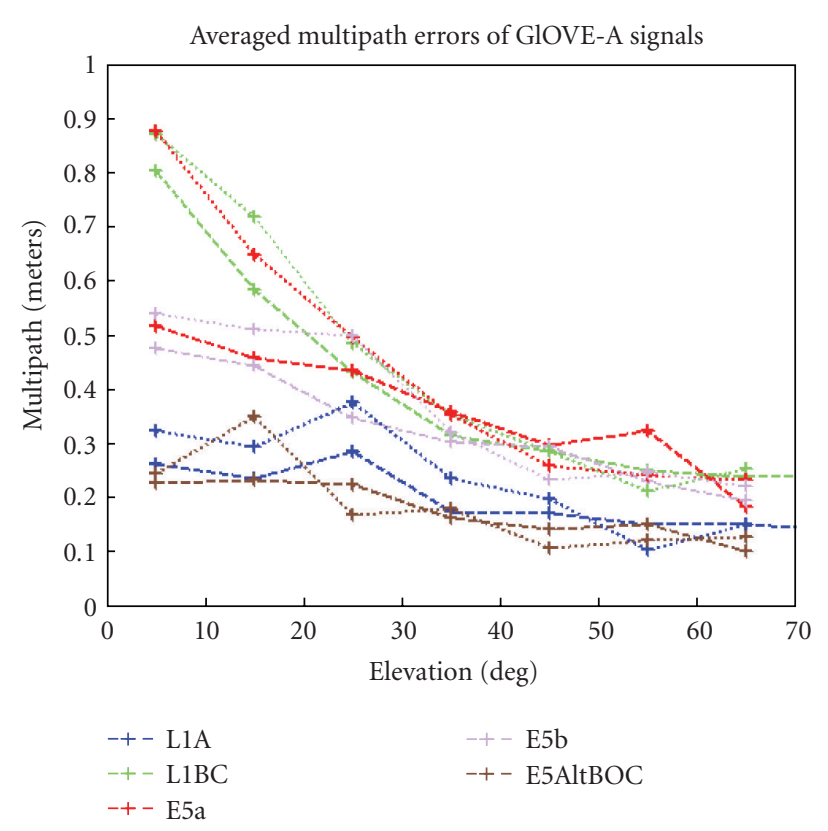

Figure 12: Dotted line: tests of December 12 and 13 at a "multipath-rich" site. Broken line: average of tests during MarchOctober, 2006, at the open-sky site.

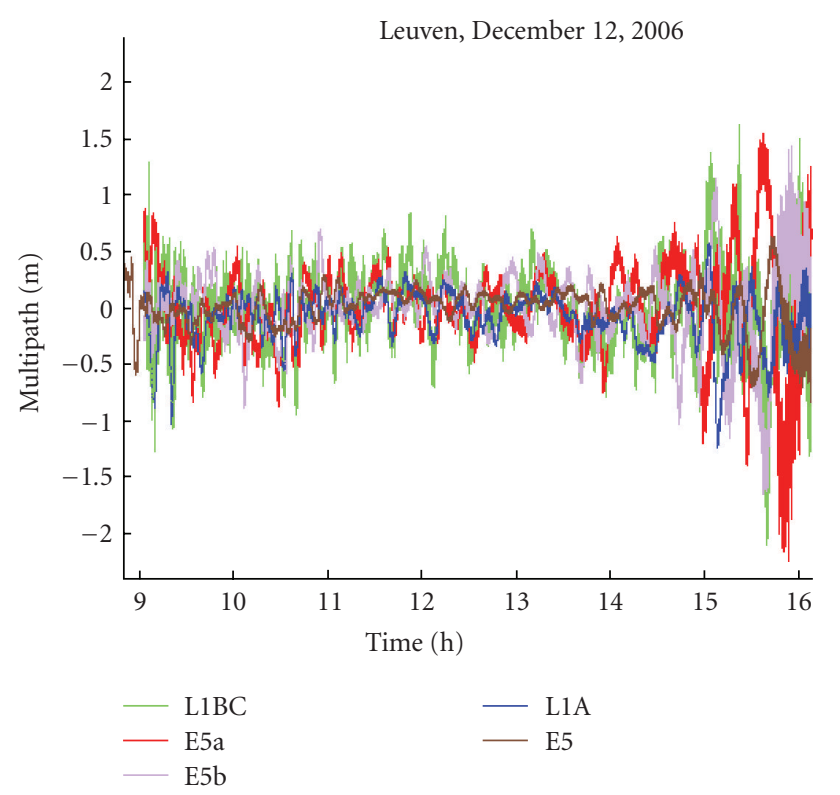

Figure 13: Time series for code multipath for December 12, 2006.

than L1A and E5AltBOC. At low elevations it still gravitates to the "high-performance group," while at higher elevations it shows about the same average multipath errors than other signals.

It is also quite clear that performance of GPS-CA on both sites is about the same as the performance of L1BC. This can probably be attributed to the prevalence of the multipath delays shorter than $150 \mathrm{~m}$, in which case both modulations are supposed to be about equivalent. The strange behavior of

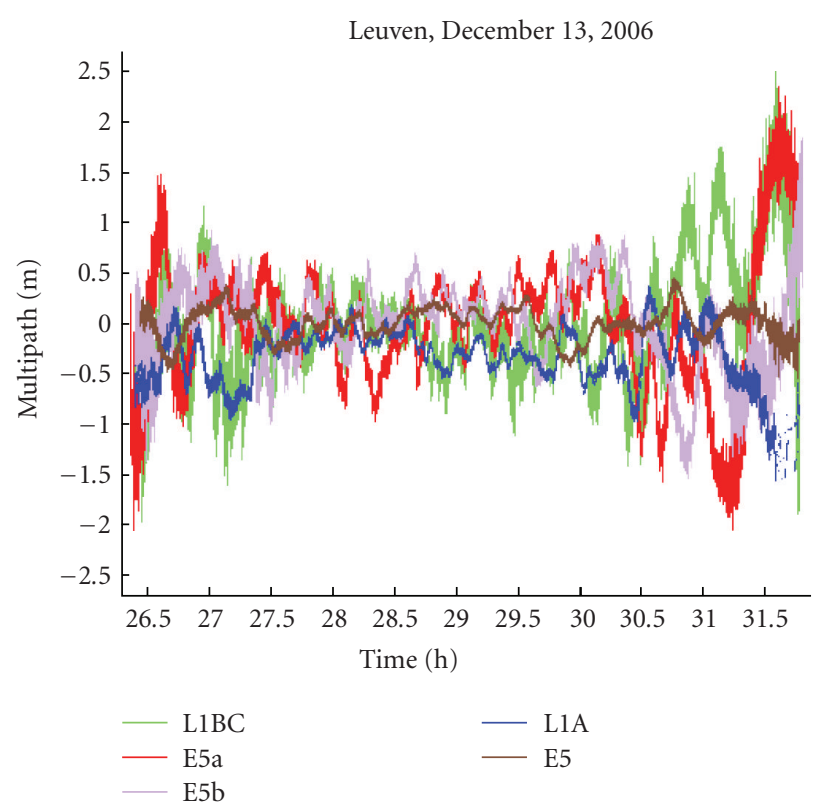

FIgURE 14: Time series for code multipath for December 13, 2006.

TABLe 3: Multipath STD error ( $\mathrm{m}$ ) of Galileo signals for a more "multipath-rich" antenna site.

\begin{tabular}{lccccc}
\hline \multirow{2}{*}{ Signal } & Chip rate & \multicolumn{2}{c}{ Dec. 12} & \multicolumn{2}{c}{ Dec. 13 } \\
& $(\mathrm{MHz})$ & $>10^{\circ}$ & $<10^{\circ}$ & $>10^{\circ}$ & $<10^{\circ}$ \\
\hline L1BC & 1.023 & 0.33 & 0.48 & 0.48 & 0.96 \\
\hline E5a & 10.23 & 0.35 & 0.82 & 0.45 & 0.89 \\
E5b & 10.23 & 0.31 & 0.32 & 0.39 & 0.60 \\
\hline L1A & 2.5575 & 0.23 & 0.20 & 0.28 & 0.36 \\
AltBOC & 10.23 & 0.16 & 0.30 & 0.21 & 0.22 \\
\hline
\end{tabular}

GPS multipath at elevations less than 10 degrees for the La Plata site can at least partly be explained by high masking angles from a wide range of directions which leads to the lower than normal availability of GNSS signals (see photo of the La Plata site, Figure 25).

Peculiarities of these sites can also be illustrated by the time series of multipath errors (Figure 17-20). Figure 17 illustrates relatively high multipath errors for E6BC. Figures 19 and 20 show that the multipath for the Wuhan stations has indeed atypical elevation dependence; at lower elevations the frequency of the variations of multipath are increasing, while their amplitude remains the same. The multipath results for different stations depend of course upon the peculiarities of the multipath environment, in particular upon the presence of reflectors oriented in a certain way relative to the GIOVEA lines of sight at its rising and setting.

Figure 21 demonstrates how different the multipath environments at different stations indeed are. At La Plata station, the multipath is generally the highest (almost a double at high elevations compared to Leuven), while at Wuhan the multipath is not only higher in general, but also its elevation dependence is flatter. Logically enough, the 
TABle 4: Availability of GIOVE-A signals for the data sets from La Plata and Wuhan.

\begin{tabular}{|c|c|c|c|c|c|c|c|c|c|}
\hline Stations & Date & $\begin{array}{l}\text { Max. } \\
\text { elevation } \\
(\mathrm{deg})\end{array}$ & L1A & L1BC & E5A & E5B & E5AltBoC & E6A & E6BC \\
\hline \multirow{2}{*}{ La Plata } & $10 \& 11$ Sep 2006 & 88.9 & $\mathrm{x}$ & $\mathrm{x}$ & & & & $\mathrm{x}$ & $\mathrm{x}$ \\
\hline & 5, $6 \& 7$ Apr 2007 & 87.7 & $\mathrm{x}$ & $\mathrm{x}$ & $\mathrm{x}$ & $\mathrm{x}$ & $\mathrm{x}$ & & \\
\hline \multirow{2}{*}{ Wuhan } & $18 \& 19$ Oct 2006 & 88.5 & $\mathrm{x}$ & $\mathrm{x}$ & & & & $\mathrm{x}$ & $\mathrm{x}$ \\
\hline & $20 \& 21$ Mar 2007 & 88.8 & & $\mathrm{x}$ & $\mathrm{x}$ & $\mathrm{x}$ & $\mathrm{x}$ & & \\
\hline
\end{tabular}

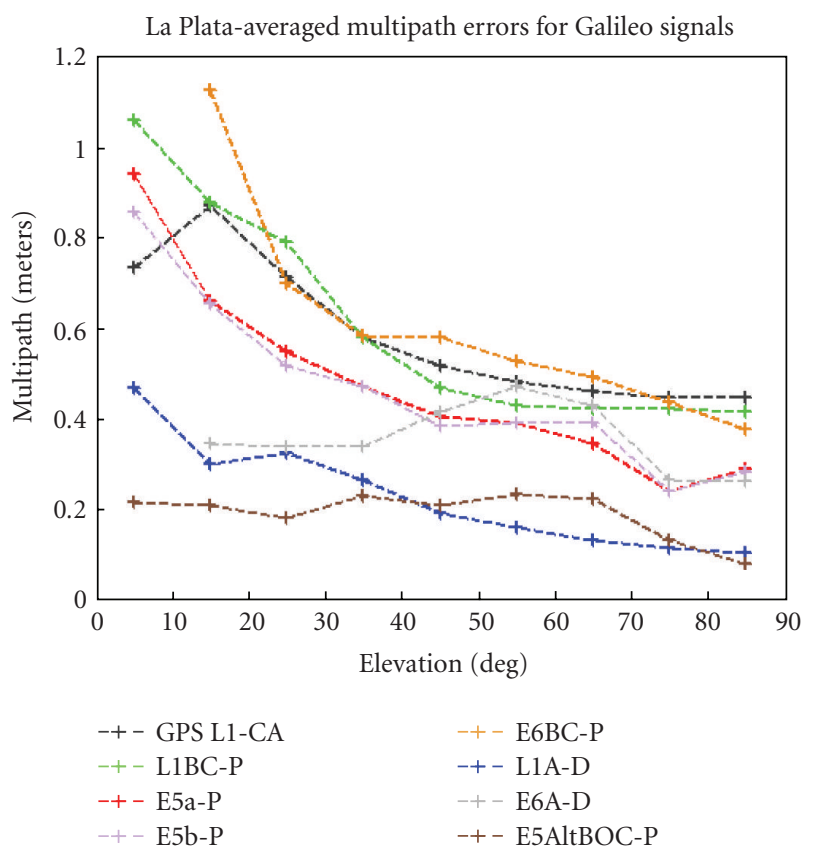

FIGURE 15: Multipath performance at the La Plata GESS site.

biggest differences can be seen for L1BC, where multipath errors are the highest, while for E5AltBOC, where multipath errors are significantly suppressed, the differences are almost undetectable (Figure 22).

Investigation of the reasons for site-dependent differences is beyond the scope of this paper. The photos of $\mathrm{La}$ Plata and Wuhan antenna sites from public IGS sources show significant amount of local reflectors. The La Plata site (Figure 25) resembles a park and is surrounded with high trees which are apparently responsible for high multipath and masking of the signal at low elelvations. The Wuhan site (Figure 26) is on the rooftop of a two-storeyed building and is surrounded by remote trees which are likely to serve as a source of scattered signals. The multipath caused by scattered signals is expected to be present at all the elevations and may be responsible for the flatter elevation dependence of multipath at Wuhan (Figure 21). It should also be mentioned that at the La Plata site the signal power is systematically lower than in Leuven and Wuhan (cf. Figures 3, 23, and 24).

The total statistics of multipath for all the processed data for La Plata and Wuhan is presented in Table 5. The

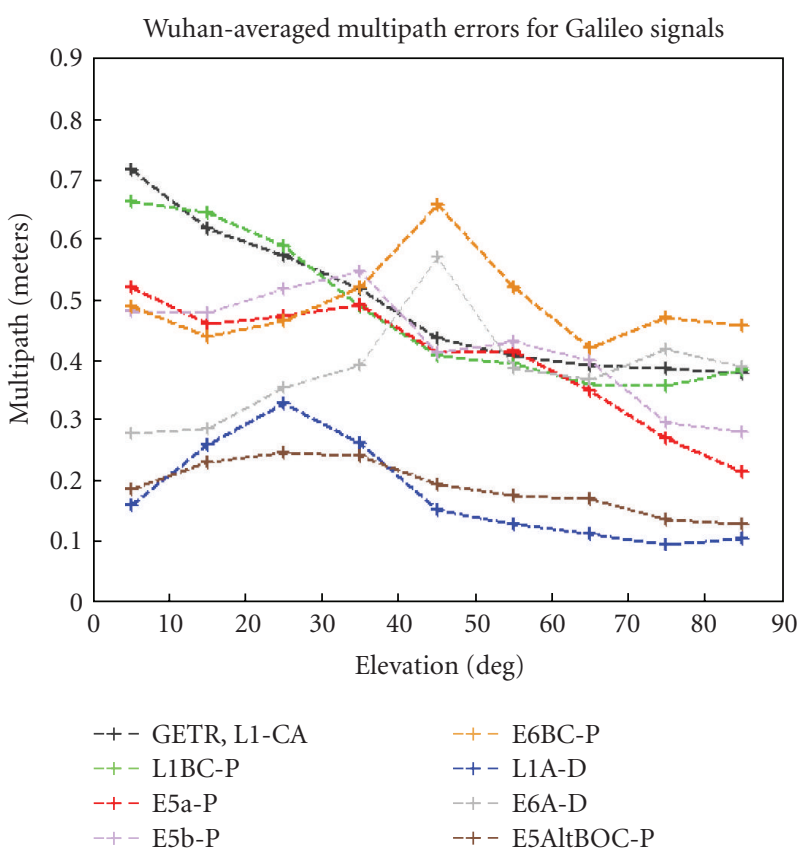

FIgURE 16: Multipath performance at the Wuhan GESS site.

averages presented in this table illustrate the same tendencies already visible from the plots, in particular the low elevation dependence of multipath at the Wuhan site.

\section{KINEMATIC TESTS}

The code multipath errors for kinematic tests with GIOVE-A signals where first presented in [1]. The kinematic multipath is very different from a static one in that its variations are dominated by fast changes of the reflectors due to movement, and that a high degree of multipath suppression is achieved at the tracking level due to averaging of the rapid oscillations of in-phase/out-of-phase multipath. The time series of kinematic multipath consists of random structure-less variations, where the differences between the modulations are much less pronounced that in the static case.

In this paper, we present the results of two car tests performed in different environments: rural and urban. Separate statistics was computed for the periods when the car was static and the periods when the car was moving. As shown in Table 6, the signal availability during the tests 
TABLE 5: Multipath 1-sigma error (m) for the data sets of La Plata/Wuhan.

\begin{tabular}{|c|c|c|c|c|c|c|c|c|c|}
\hline \multirow{3}{*}{ Signal } & \multirow{3}{*}{$\begin{array}{c}\text { Chip } \\
\text { rate } \\
(\mathrm{MHz})\end{array}$} & \multicolumn{4}{|c|}{ La Plata } & \multicolumn{4}{|c|}{ Wuhan } \\
\hline & & \multicolumn{2}{|c|}{ Sep. 10} & \multicolumn{2}{|c|}{ Apr. 05} & \multicolumn{2}{|c|}{ Oct. 18} & \multicolumn{2}{|c|}{ Mar. 20} \\
\hline & & $>10^{\circ}$ & $<10^{\circ}$ & $>10^{\circ}$ & $<10^{\circ}$ & $>10^{\circ}$ & $<10^{\circ}$ & $>10^{\circ}$ & $<10^{\circ}$ \\
\hline L1BC & 1.023 & 0.56 & - & 0.62 & 1.06 & 0.53 & 0.66 & 0.54 & 0.66 \\
\hline E5a & 10.23 & - & - & 0.46 & 0.94 & - & - & 0.42 & 0.52 \\
\hline $\mathrm{E} 5 \mathrm{~b}$ & 10.23 & - & - & 0.46 & 0.86 & - & - & 0.46 & 0.48 \\
\hline E6BC & 5.115 & 0.59 & - & - & - & 0.50 & 0.49 & - & - \\
\hline L1A & 2.5575 & 0.28 & - & 0.28 & 0.47 & 0.29 & 0.16 & - & - \\
\hline E6A & 5.115 & 0.38 & - & - & - & 0.39 & 0.28 & - & - \\
\hline AltBOC & 10.23 & - & - & 0.21 & 0.22 & - & - & 0.21 & 0.19 \\
\hline
\end{tabular}

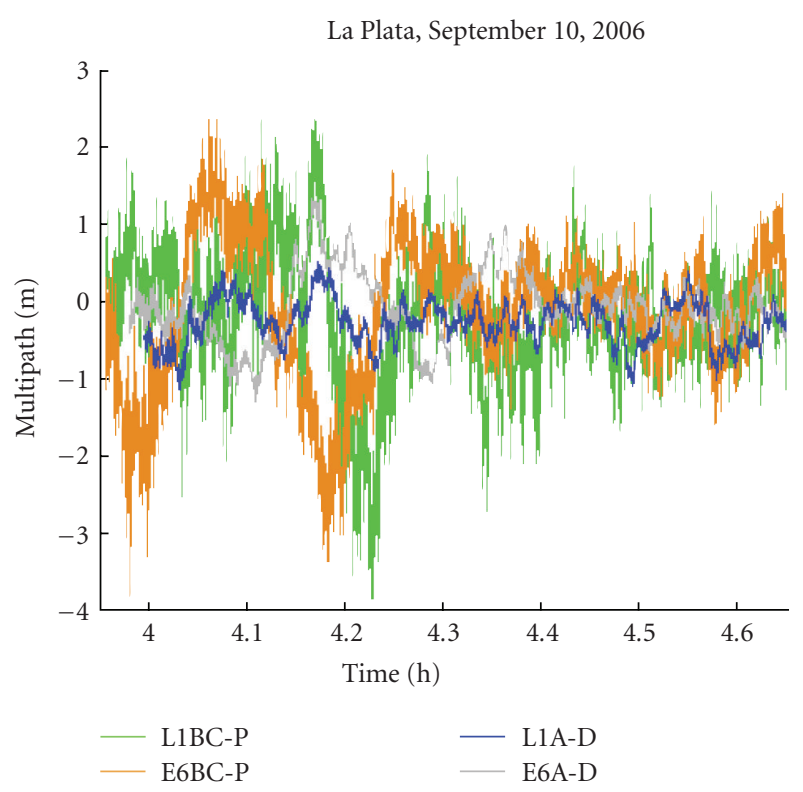

Figure 17: Time series of code multipath for La Plata, September 10, 2006.

was different; during the urban test, L1 and E6 were being transmitted, while during the rural test L1 and E5 signals were available.

Although the static portions of the car tests still show the same tendencies as the data collected on the rooftop, the data collected during the movement demonstrates much smaller values of multipath errors, much smaller advantage of Galileo modulations as compared to GPS-C/A, and much smaller differences between Galileo modulations. The differences between static and kinematic multipath can be clearly seen in Figures 27 and 28. Figure 29 illustrates that code multipath during the urban test was generally somewhat higher due to obviously greater amount of reflectors in the urban environment.

In particular, the results of the car tests suggest that the replacement of $\mathrm{L} 1 \mathrm{BOC}(1,1)$ with $\mathrm{MBOC}$ will not have any significant impact on the multipath performance in

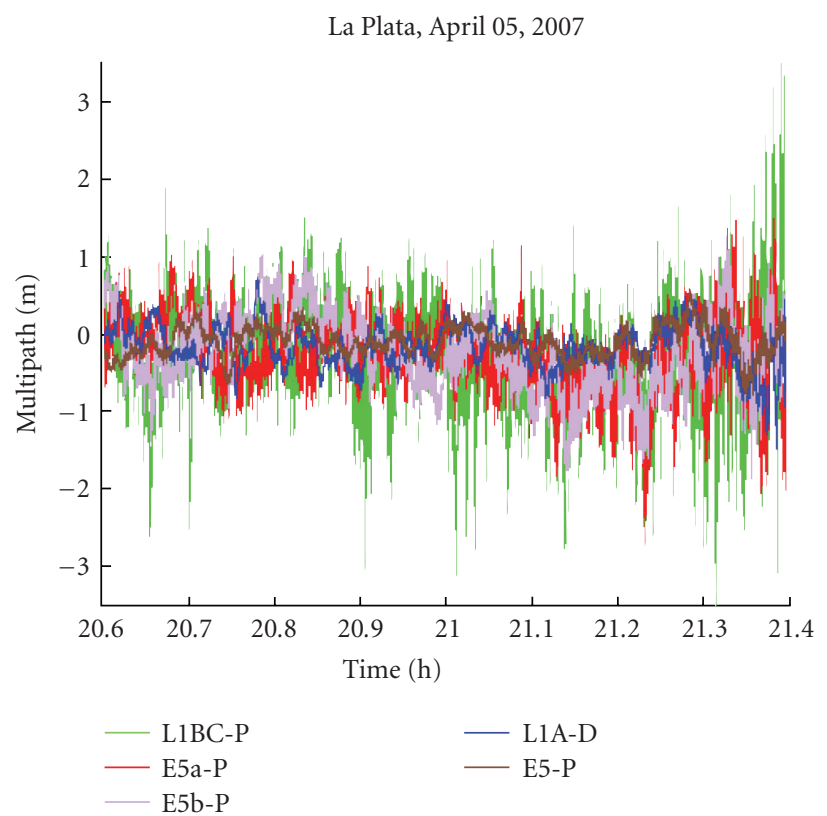

Figure 18: Time series of code multipath for La Plata, April 05, 2007.

the automotive environment. Indeed, MBOC is expected to show the performance intermediate between L1BC and E6BC, while both modulations have about the same intensity of kinematic multipath according to Table 6 .

According to theory, MBOC is expected to outperform $\operatorname{BOC}(1,1)$ for static scenarios, possibly bringing greater improvement relative to $\operatorname{BOC}(1,1)$ than the improvement of the $\operatorname{BOC}(1,1)$ relative to $\operatorname{BPSK}(1)$. This is to be verified after actual implementation of MBOC.

\section{PHASE MULTIPATH}

Simultaneous availability of 3 frequencies allows direct evaluation of phase multipath from triple-frequency ionofree geometry-free combinations of phase measurements $[1,9]$ :

$$
M_{\Phi 123}=\lambda_{3}^{2}\left(\Phi_{1}-\Phi_{2}\right)+\lambda_{2}^{2}\left(\Phi_{3}-\Phi_{1}\right)+\lambda_{1}^{2}\left(\Phi_{2}-\Phi_{3}\right) .
$$


TABLE 6: Multipath statistic for car tests (m).

\begin{tabular}{lcccc}
\hline & Rural static & Rural movement & Urban static & Urban movement \\
\hline GPS-CA & & & 1.19 & 0.23 \\
L1BC & 0.27 & 0.15 & 0.40 & 0.18 \\
E6BC & & & & 0.22 \\
E5a & 0.20 & 0.16 & & \\
E5b & 0.26 & 0.15 & & \\
E5AltBOC & 0.10 & 0.11 & & \\
\hline
\end{tabular}

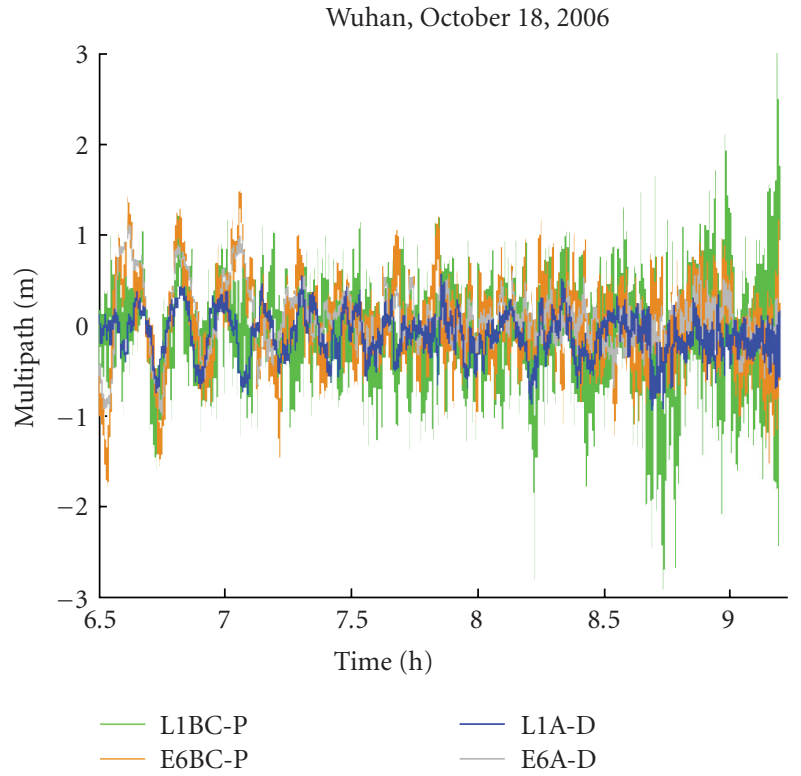

FIGURE 19: Time series of code multipath for Wuhan, October 18, 2006.

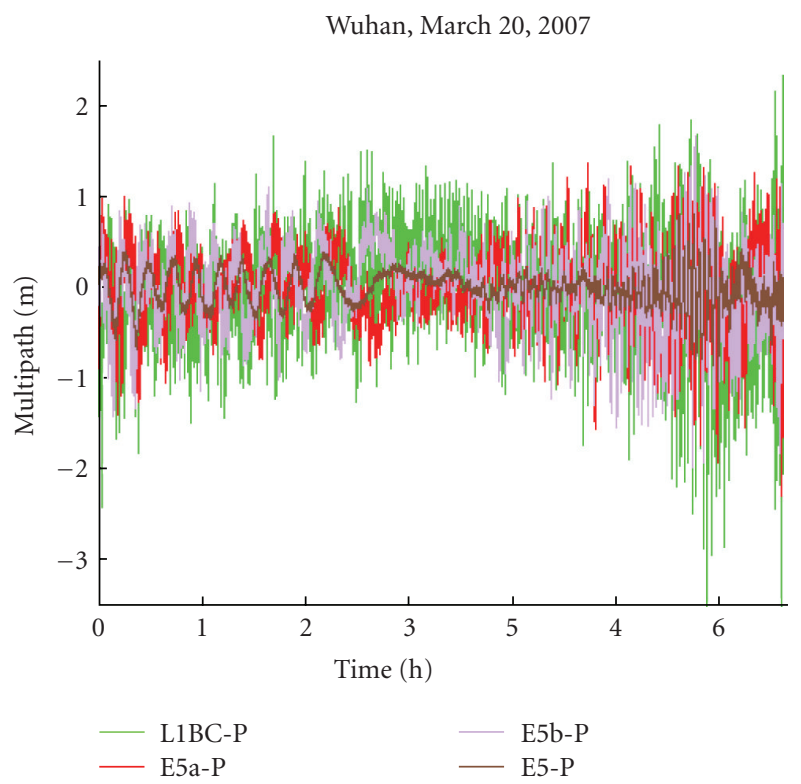

FIgure 20: Time series of code multipath for Wuhan, March 20, 2007.

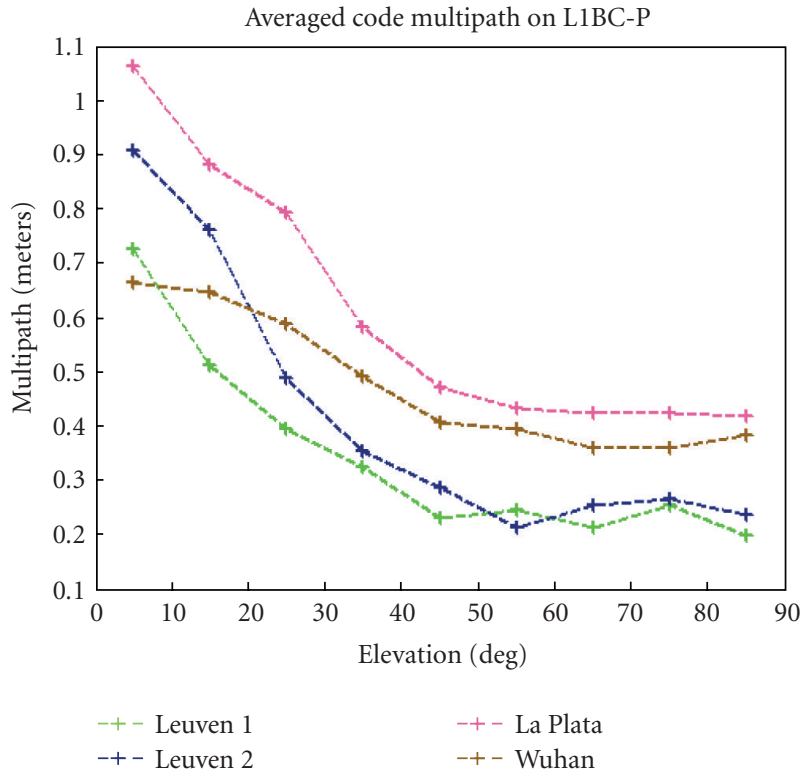

FIgure 21: Code multipath on L1BC at 4 locations. Here "Leuven1 " is our main open-sky antenna site (Figure 2). "Leuven-2" is more multipath-rich site located between the ventilation outlets (see Section 5).

This formula is a linear combination of three geometry-free observables $\left(\Phi_{i}-\Phi_{j}\right)$, which all contain ionosphere delays. As it has been shown in [9], in (2) ionosphere delays cancel out. $M_{123}$ contains a mix of phase multipath and tracking errors for the same satellite on 3 different frequencies and can be used as a global indicator of phase multipath severity. It can be used in particular to study elevation dependence and site dependence of phase multipath.

In this paper, we used one particular combination (E5a $-1.128^{*} \mathrm{E} 5 \mathrm{~b}+0.128^{*} \mathrm{~L} 1 \mathrm{BC}$ ) as an indicator of phase multipath. Figure 30 contains elevation dependence of this phase multipath indicator for all the static sites covered in this paper. The elevation dependence shows significant variability and does not indicate with certainty any differences between the sites.

The nature of phase multipath is in general quite different from code multipath. In particular, phase multipath for different signals is not expected to show significant differences. It has already been demonstrated in [1] that the phase tracking noise is identical for all the GIOVE-A signals. 


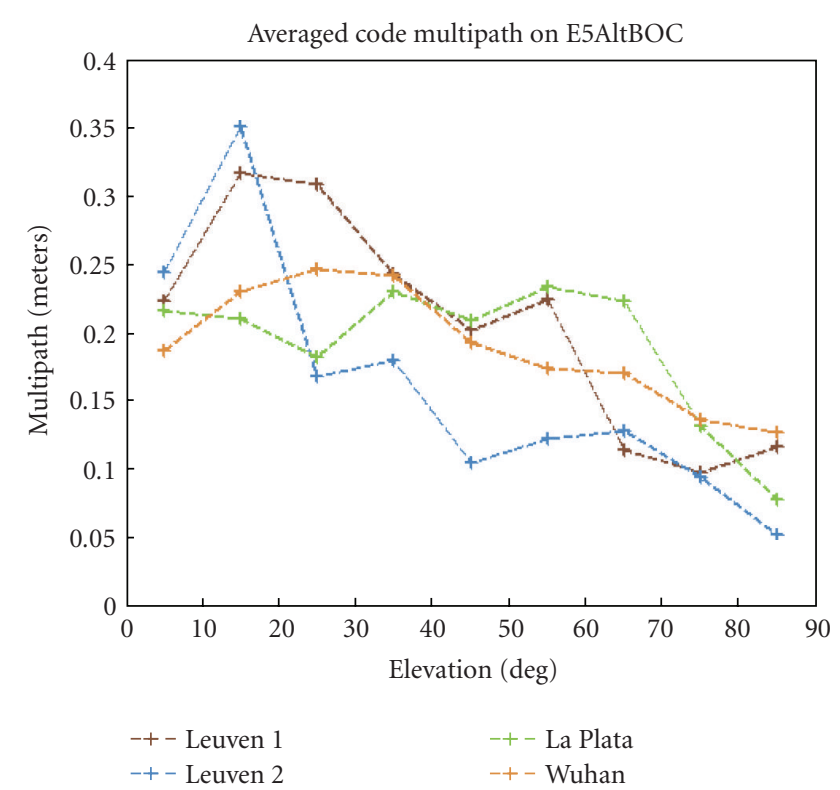

Figure 22: Code multipath on E5AltBOC at 4 locations. Here "Leuven-1" is our main open-sky antenna site (Figure 2). "Leuven2 " is more multipath-rich site located between the ventilation outlets (see Section 5).

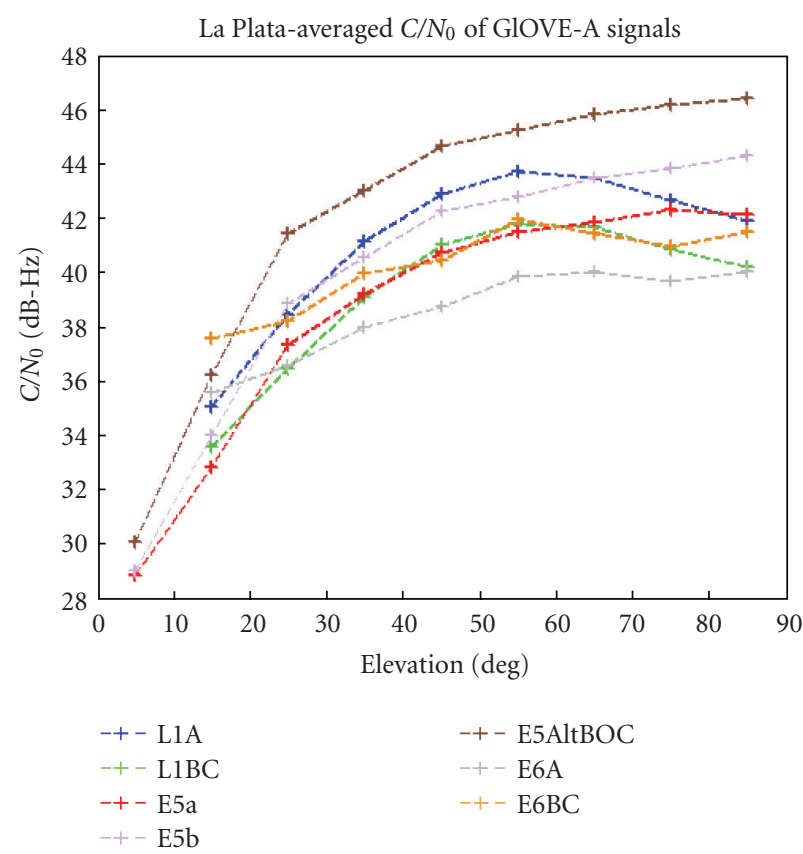

Figure 23: Signal power at La Plata station. It is systematically lower as compared to Leuven (Figure 3) and Wuhan (Figure 24).

Phase multipath is generally much less studied than code multipath, so it is difficult to predict what the behavior of phase multipath should be. The time series of our phase multipath indicator is presented in Figure 31.

The elevation dependence of phase multipath is generally flatter and more variable that with the code multipath. There

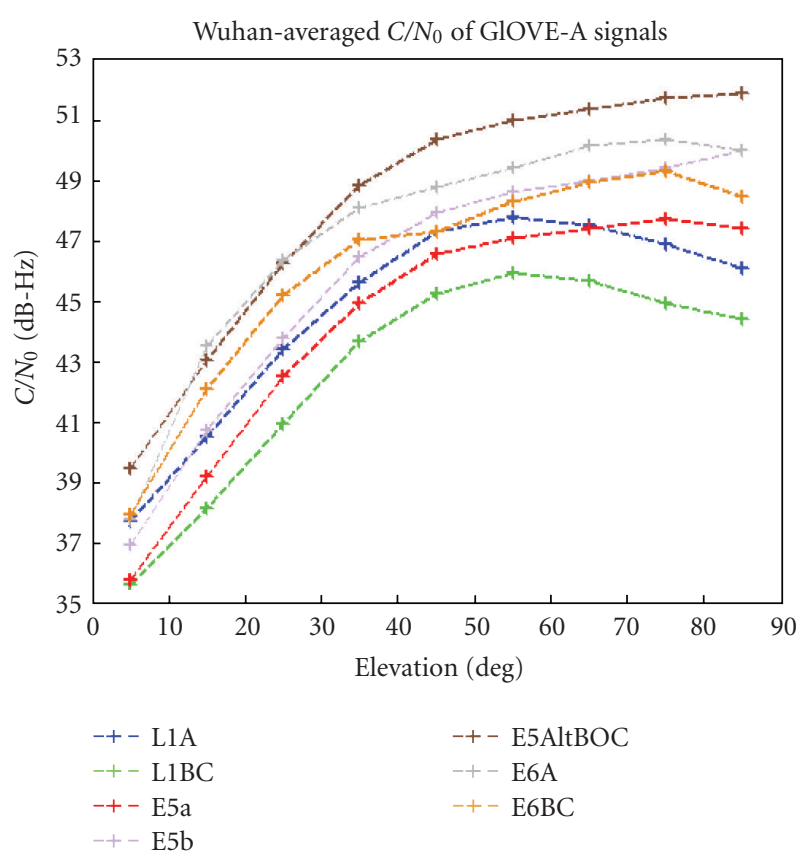

FIGURE 24: Signal power at Wuhan station.

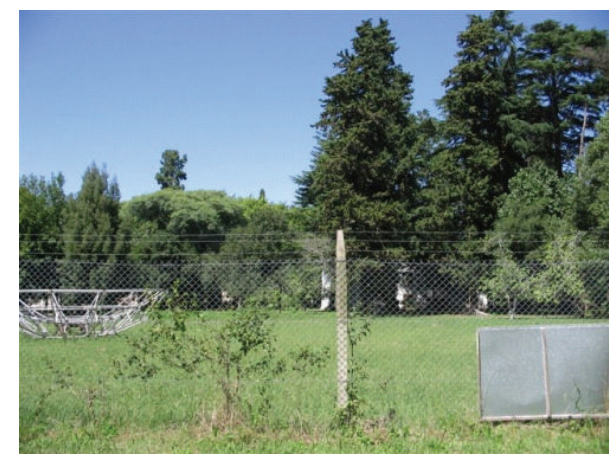

Figure 25: Environment at the La Plata antenna site.

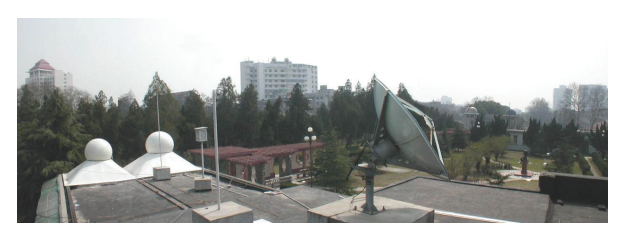

FIGURE 26: Environment at the Wuhan antenna site.

exist significant long-term variations which have impact on the statistics in a way of making it less stable. The pattern of phase multipath is quite different between the sites (cf. Figures 31 and 32).

\section{CONCLUSIONS}

Field experience with GIOVE-A signals has demonstrated stable reception in a variety of external conditions and 


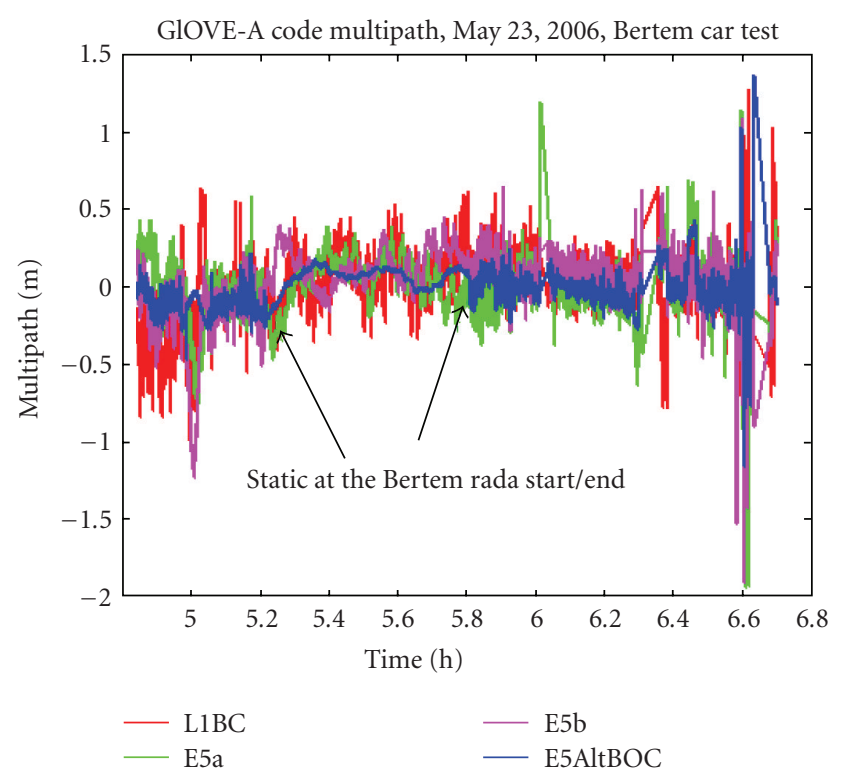

FIGURE 27: Code multipath during the rural car test.

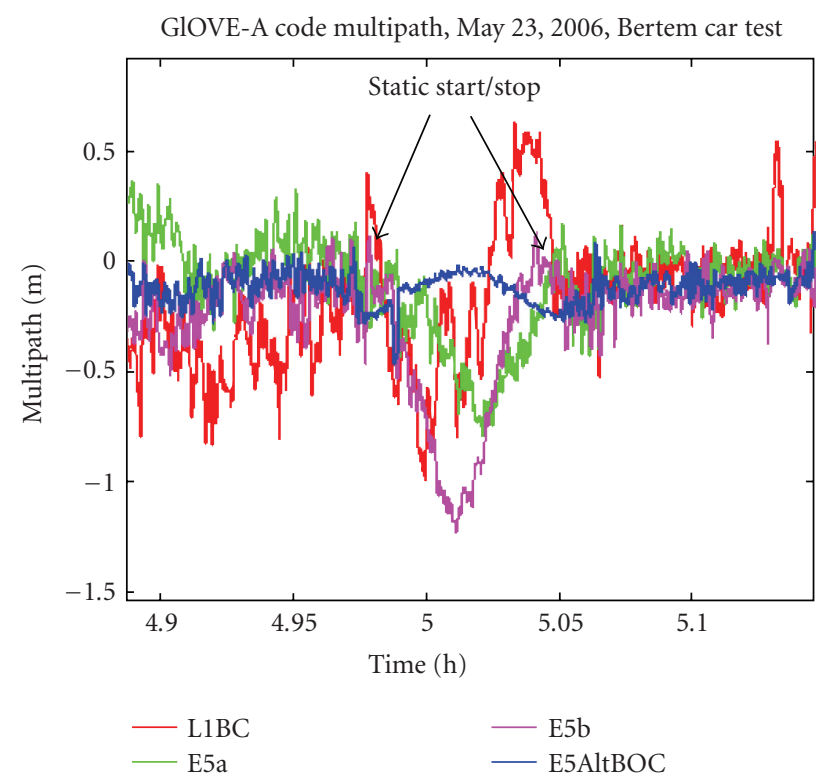

FIGURE 28: Zoom into one of the static portions of the rural test.

confirmed the theoretical expectations as to superior multipath rejection of wide-band Galileo modulations. Multipath performance results for static and kinematic tests have been reported.

Comparison of the static data from different sites shows significant variability of the multipath performance for most of the Galileo signals. It seems that only the behavior of E5AltBOC is truly stable and repeatable for all the tests; in all the tests, the E5AltBOC demonstrates the highest multipath suppression as compared to other signals and very

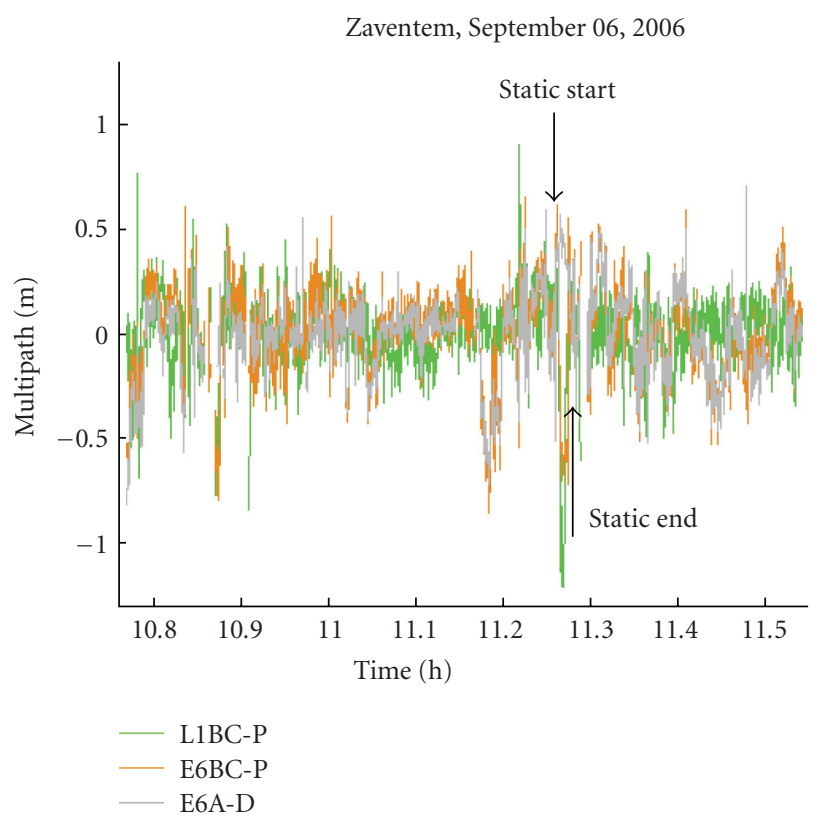

Figure 29: Code multipath during the urban test.

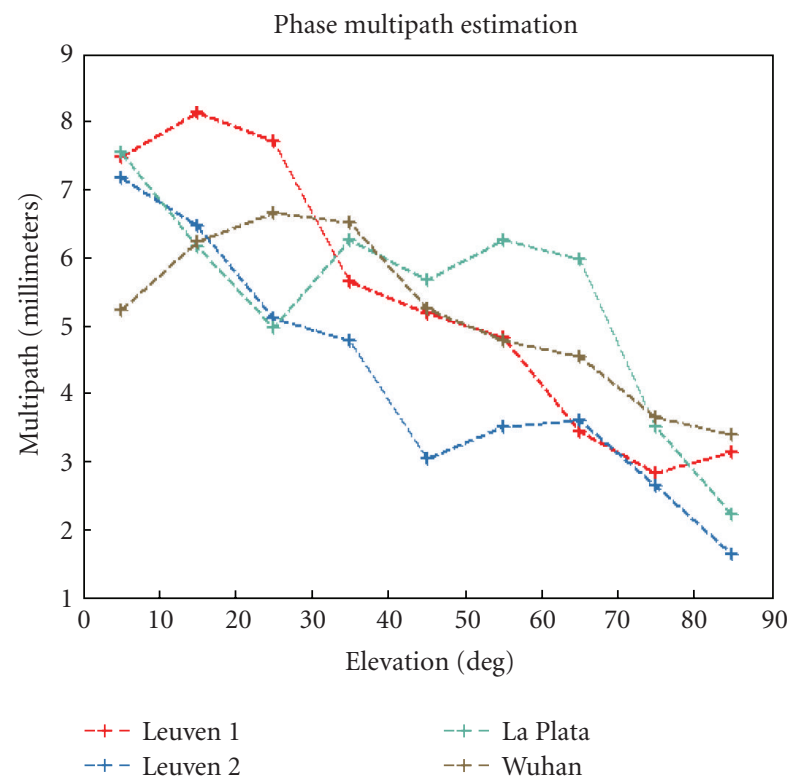

Figure 30: Phase multipath at 4 locations. Here "Leuven-1" is our main open-sky antenna site (Figure 2). "Leuven-2" is a more multipath-rich site located between the ventilation outlets (see Section 7).

low magnitude of average multipath errors, down to the values about $0.2 \mathrm{~m}$.

For all the other signals, we can talk about the tendencies which manifest themselves on average, but with significant site-dependent variations. The most important of these tendencies is the classification of all the modulations in groups shown in Table 2. According to this classification, $\mathrm{E} 6 \mathrm{~A}+\mathrm{L} 1 \mathrm{~A}+\mathrm{E} 5 \mathrm{AltBOC}$ form the group of high-performance 


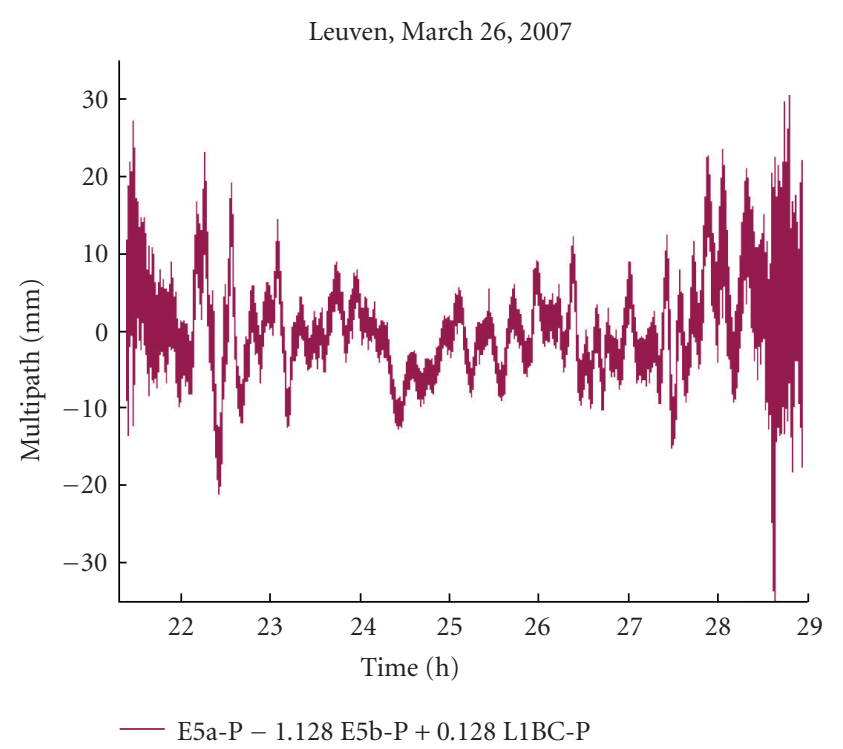

FIgUre 31: Phase multipath indicator (triple-frequency phase combination of L1BC, E5a, and E5b) at Leuven site.

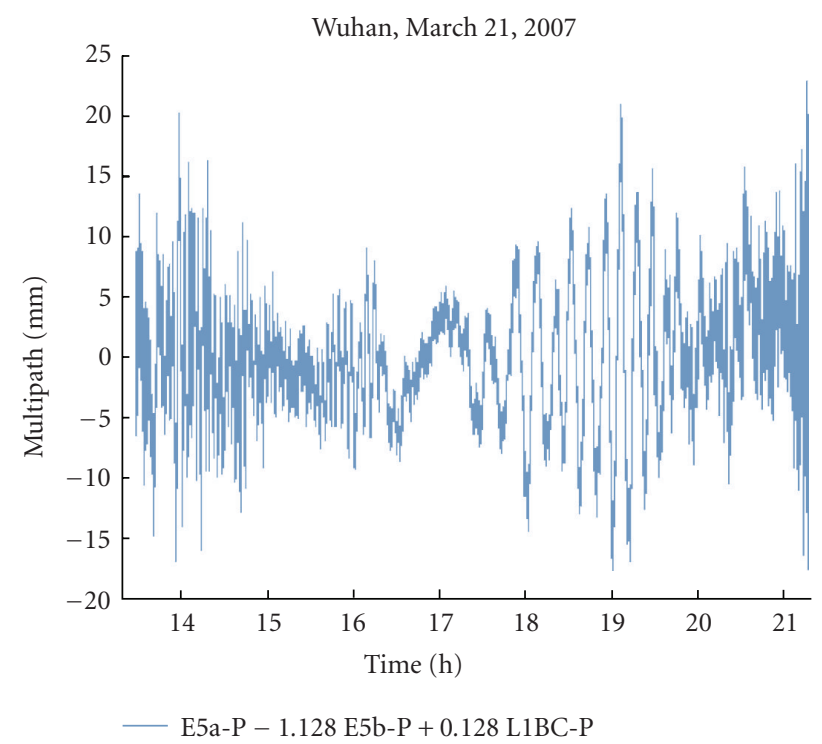

FIgURE 32: Phase multipath indicator (triple-frequency phase combination of L1BC, E5a, and E5b) at Wuhan.

signals, while the $\mathrm{E} 5 \mathrm{a}, \mathrm{E} 5 \mathrm{~b}$, and $\mathrm{E} 6 \mathrm{BC}$ signals belong to the medium group, the performance of $\mathrm{L} 1 \mathrm{BC}$ is the lowest.

This classification, which agrees with theoretical predictions and computer simulations, can be accepted as a general rule, although in some tests E6BC and E5a,b show practically the same performance as L1BC, and E6A in the others shows performance more typical to the medium group.

The relationship between the signals for individual sites depends upon the spectra of multipath delays. For the Leuven site where the long-range multipath with a delay of about $200 \mathrm{~m}$ is clearly dominant, the wide-band signals with essential suppression of long-range multipath component show clearly superior performance. In other cases, when short-range multipath is dominant, the advantage of more advanced codes will be less pronounced.

The future research may take an approach of looking in more detail into specific multipath conditions and types of reflectors at different sites. Accumulation of much greater statistic may help to formulate the trends in a more reliable and detailed manner and make a classification of sites in accordance with the multipath behavior.

The kinematic tests have demonstrated a lot of smaller values of multipath errors and a much less significant dependence of multipath upon code modulations. This means in particular that any further changes in the signal definition of Galileo signals are not likely to bring any significant improvement to dynamic applications, such as automotive, although modulation changes may have impact on static applications.

In this paper, the phase multipath statistics for GIOVE-A signals is presented for the first time.

\section{ACKNOWLEDGMENT}

The authors would like to thank M. Falcone for his support of GIOVE signal experimentation activity.

\section{REFERENCES}

[1] A. Simsky, J.-M. Sleewaegen, M. Hollreiser, and M. Crisci, "Performance assessment of Galileo ranging signals transmitted by GSTB-V2 satellites," in Proceedings of the 19th International Technical Meeting of the Satellite Division of the Institute of Navigation (ION GNSS '06), vol. 3, pp. 1547-1559, Fort Worth, Tex, USA, September 2006.

[2] M. Hollreiser, M. Crisci, J.-M. Sleewaegen, et al., "Galileo signal experimentation," GPS World, vol. 18, no. 5, pp. 44-50, 2007.

[3] M. Spelat, M. Crisci, M. Falcone, and M. Hollreiser, "GIOVEA signal in space test activity at ESTEC," in Proceedings of the 19th International Technical Meeting of the Satellite Division of the Institute of Navigation (ION GNSS '06), vol. 2, pp. 981-983, Fort Worth, Tex, USA, September 2006.

[4] M. Falcone, M. Lugert, M. Malik, et al., "GIOVE-A in orbit testing results," in Proceedings of the 19th International Technical Meeting of the Satellite Division of the Institute of Navigation (ION GNSS '06), vol. 3, pp. 1535-1546, Fort Worth, Tex, USA, September 2006.

[5] M. Hollreiser, J.-M. Sleewaegen, W. de Wilde, M. Falcone, and F. Wilms, "Galileo test user segment," GPS World, vol. 16, no. 7, pp. 23-29, 2005.

[6] W. de Wilde, J.-M. Sleewaegen, A. Simsky, et al., "New fast signal acquisition unit for GPS/Galileo receivers," in Proceedings of the European Navigation Conference (ENC GNSS '06), Manchester, UK, May 2006.

[7] J.-M. Sleewaegen, W. de Wilde, and M. Hollreiser, "Galileo Alt-BOC receiver," in Proceedings of the European Navigation Conference (GNSS '04), Rotterdam, The Netherlands, May 2004.

[8] A. Fernandez, J. Diez, C. Griffin, et al., "UERE budget results for the Galileo test user receiver," in Proceedings of the 19th International Technical Meeting of the Satellite Division of the Institute of Navigation (ION GNSS '06), vol. 2, pp. 1048-1059, Fort Worth, Tex, USA, September 2006.

[9] A. Simsky, "Three's the charm: triple-frequency combinations in future GNSS," Inside GNSS, vol. 1, no. 5, pp. 38-41, 2006. 

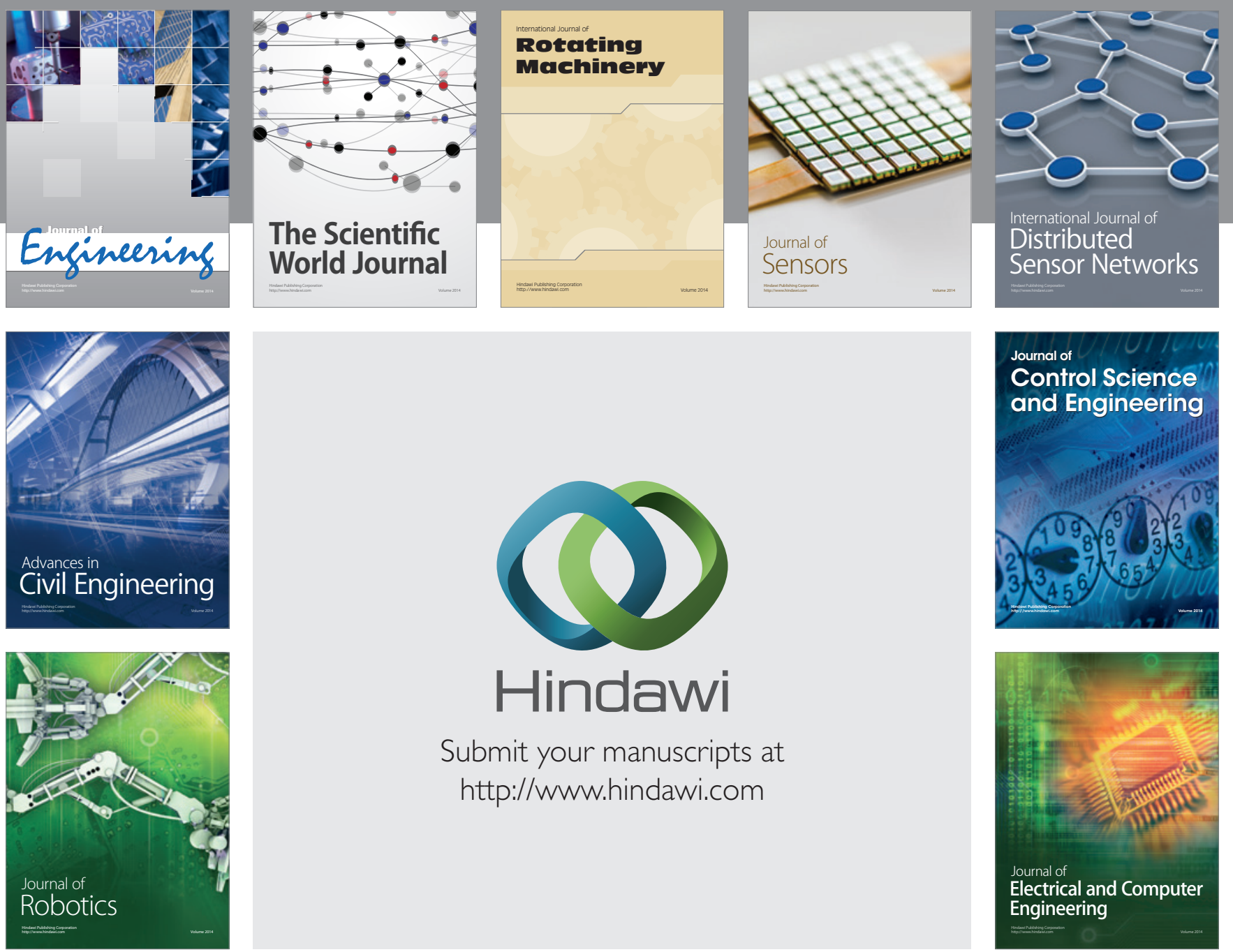

Submit your manuscripts at

http://www.hindawi.com
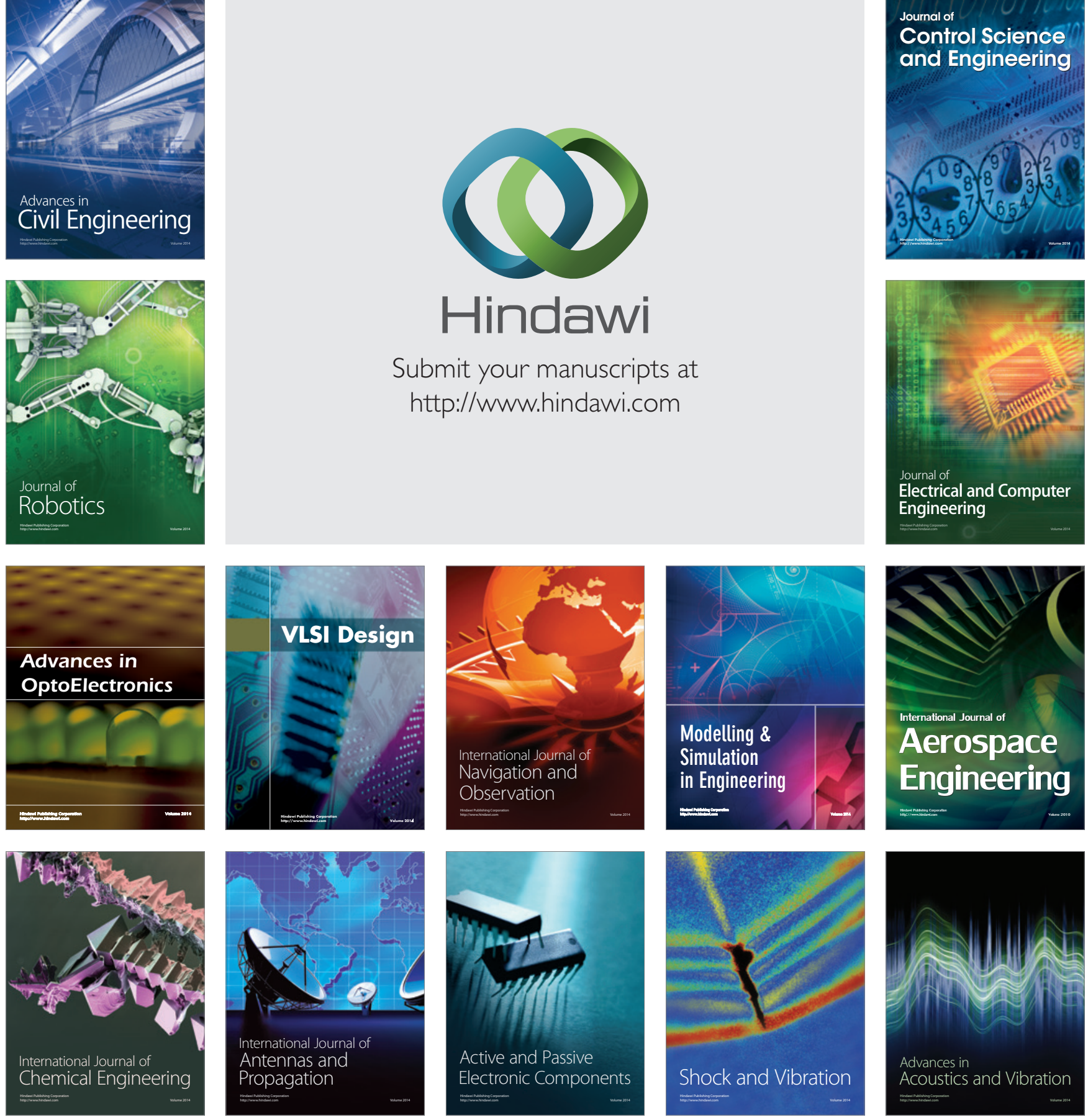